\title{
Lhx2 in germ cells suppresses endothelial cell migration in the developing ovary
}

Neha Singh ${ }^{1}$, Domdatt Singh ${ }^{1}$, Anshul Bhide ${ }^{1}$ Richa Sharma $^{1}$ Sarthak Sahoo ${ }^{2}$, Mohit Kumar Jolly $^{2}$ and Deepak Modi ${ }^{*}$

${ }^{1}$ Molecular and Cellular Biology Laboratory, ICMR-National Institute for Research in Reproductive and Child Health, Indian Council of Medical Research (ICMR), JM Street, Parel, Mumbai 400012, India

${ }^{2}$ Center for BioSystems Science and Engineering, Indian Institute of Science, CV Raman Rd, Bangalore, 560012, India

Orcid ID:

Neha Singh (Orcid ID: 0000-0001-7088-839X)

Domdatt Singh (Orcid ID: 0000-0002-3361-0388)

Anshul Bhide (Orcid ID: 0000-0003-4592-7451)

Richa Sharma (Orcid ID: 0000-0001-8490-9761)

Sarthak Sahoo (Orcid ID: 0000-0002-7771-5894)

Mohit Kumar Jolly (Orcid ID: 0000-0002-6631-2109)

Deepak Modi (Orcid ID: 0000-0002-4230-4219)

\section{Running Title: Lhx2 suppresses mesonephric cell migration into the ovary}

* Corresponding author

Dr. Deepak Modi

Scientist $F$ and Head

Molecular and Cellular Biology Laboratory

ICMR-National Institute for Research in Reproductive and Child Health

Indian Council of Medical Research (ICMR)

J.M. Street, Parel'

Mumbai 400012

India

Tel no: 91-22-24192034

Fax no: 91-22-24139412

Email: deepaknmodi@yahoo.com, modid@nirrh.res.in

Keywords: Homeobox, LIM gene, LIM-HD, Sex determination, Gonad development, Testis, Endothelial cell migration, Angiogenesis, Vasculature 
Wild Type Ovary High Lhx2 in germ cells

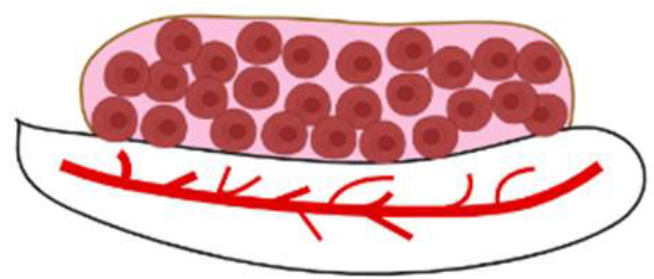

Anti-angiogenic milieu No Vascularization
Wild Type Testis Low Lhx2 in germ cells

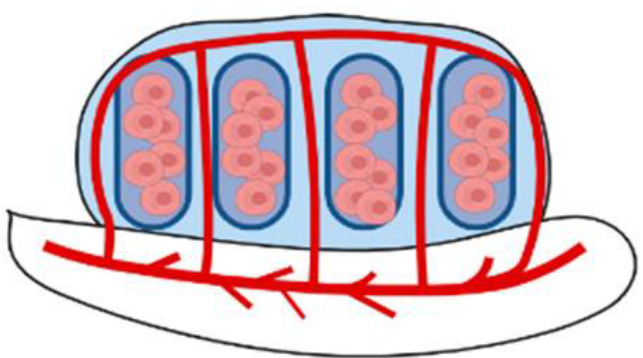

Pro-angiogenic milieu Vascularization

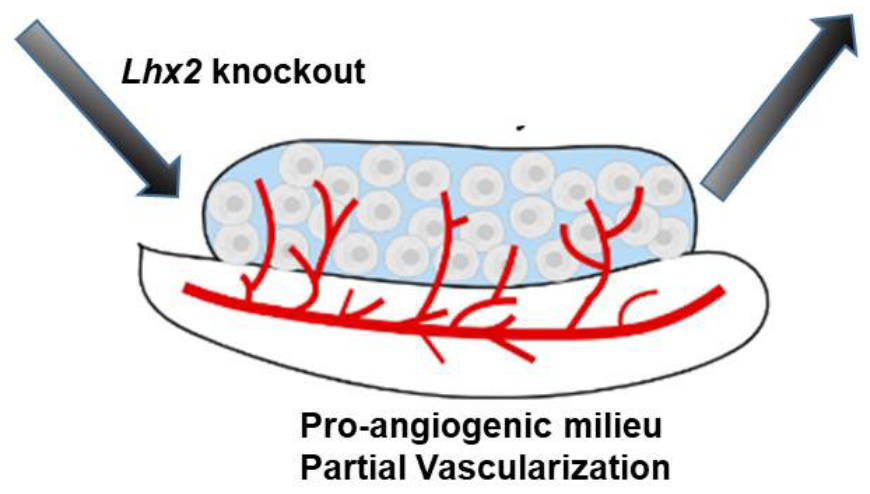

\section{Highlight}

- Multiple LIM-HD genes are expressed in developing gonads during the window of sex determination with $L h x 2$ having female dominating expression in an evolutionarily conserved manner

- Lhx2 is expressed in the germ cells of developing mouse ovaries

- Loss of $\operatorname{Lh} x 2$ in the developing ovaries alters the expression of genes involved in various pathways including angiogenesis

- Lhx2 in germ cells suppress endothelial cell migration in the developing ovaries 


\begin{abstract}
LIM-homeobox genes play multiple roles in developmental processes, but their roles in gonad development are not completely understood. Herein, we report that Lhx2, Ils2, Lmxla, and $L m x l b$ are expressed in a sexually dimorphic manner in mouse, rat, and human gonads during sex determination. Amongst these, Lhx2 has female biased expression in the developing gonads of species with environmental and genetic modes of sex determination. Single-cell RNAseq analysis revealed that Lhx2 is exclusively expressed in the germ cells of the developing mouse ovaries. To elucidate the roles of $L h x 2$ in the germ cells, we analyzed the phenotypes of Lhx2 knockout XX gonads. While the gonads developed appropriately in Lhx2 knockout mice and the somatic cells were correctly specified in the developing ovaries, transcriptome analysis revealed enrichment of genes in the angiogenesis pathway. There was an elevated expression of several pro-angiogenic factors in the Lhx2 knockout ovaries. The elevated expression of pro-angiogenic factors was associated with an increase in numbers of endothelial cells in the Lhx2-/- ovaries at E13.5. Gonad recombination assays revealed that the increased numbers of endothelial cells in the XX gonads in absence of Lhx2 was due to ectopic migration of endothelial cells in a cell non-autonomous manner. We also found that, there was increased expression of several endothelial cell-enriched male-biased genes in Lhx2 knockout ovaries. Also, in absence of $L h x 2$, the migrated endothelial cells formed an angiogenic network similar to that of the wild type testis, although the coelomic blood vessel did not form. Together, our results suggest that $L h x 2$ in the germ cells is required to suppress vascularization in the developing ovary. These results suggest a need to explore the roles of germ cells in the control of vascularization in developing gonads.
\end{abstract}




\section{Introduction}

In most sexually dimorphic species, the gonads develop as a bipotential urogenital ridge that differentiates either into the testis or an ovary depending on genetic and/or environmental cues. The coelomic epithelium from the ventral surface of mesonephros proliferates and provides the somatic niche; the primordial germ cells migrate and colonize to form the gonad. In the mouse, Sry gene is induced in the XY gonads around E10.5 that leading to the differentiation of somatic cells to Sertoli cells (Ortega et al., 2019; Rotgers et al., 2018). Under a well-defined genetic network, the Sertoli cells organize to form seminiferous tubules which is the hallmark of testicular differentiation (Nef et al., 2019; Rotgers et al., 2018; Singh and Modi, 2020; Yildirim et al., 2020). Along with somatic cell differentiation, there is sexspecific vascularization. In the XY gonads at E12.5, the mesonephric vascular plexus breaks down releasing the mesonephric endothelial cells which migrate into the testis establishing a vascular network and forming a coelomic blood vessel (Coveney et al., 2008; Svingen and Koopman, 2013; Ungewitter and Yao, 2013). Signalling factors like Fgfs, Pdgf, and Vegf aid in endothelial migration and male-specific blood vascular patterning (Cool et al., 2011; Gu et al., 2021; Svingen and Koopman, 2013). Vascularization is crucial for the delivery of exogenous factors in the testicular compartments, in the initial organization of the testis cords, and also in the regulation of testis morphogenesis (Gu et al., 2021; Singh and Modi, 2020; Svingen and Koopman, 2013). It is also required for maintaining the interstitial progenitor population of the fetal Leydig cells (Gu et al., 2021; Kumar and DeFalco, 2018; Li et al., 2021).

During development, angiogenesis is specific to the XY gonads as endothelial cell migration and vascularization do not occur in the XX gonads. Although vascular endothelial cells are detected in the fetal ovary, these do not arise from the mesonephros and neither forms the vascular network, rather the vasculature grows by pre-existing branches and its reorganization (Coveney et al., 2008). However, ectopic migration of endothelial cells from the mesonephros is detected in the fetal ovaries of mice knockout for Rspol, Wnt4, Ctnnbl, or Fst, and the endothelial cells also form a coelomic blood vessel similar to that observed in the testis (Chassot et al., 2014; Nicol and Yao, 2015; Tang et al., 2020; Yao et al., 2006). These results indicate that although a genetic program is required to initiate vascularization in the $\mathrm{XY}$ gonads, there also exists a genetic program to suppress vascularization in the developing $\mathrm{XX}$ gonads. While the biological need to suppress vascularization in the 
developing ovary is not clear, ectopic vascularization is accompanied by differentiation of Sertoli-like cells and fetal Leydig cells in the developing XX gonads (Chassot et al., 2014; Tang et al., 2020). In the testis too, disrupted vascularization promotes premature differentiation of fetal Leydig cells (Kumar and DeFalco, 2018). These observations imply that suppression of vascularization may be required to prevent masculinization of the fetal ovary. However, beyond Wnt4, Rspol, Ctnnbl, and Fst; factors (if any) that suppress vascularization in the fetal ovary are unknown.

LIM homeobox (Lhx) genes encode for transcription factors that contain two tandem $\mathrm{N}$ terminal LIM domains and centrally located a DNA binding homeodomain (HD). The LIM homeodomain (LIM-HD) proteins execute their functions through protein-protein interactions mediated by LIM domains and the homeodomain controls gene expression (Singh et al., 2021; Yasuoka and Taira, 2021). During embryonic development, LIM-HD genes are crucial regulators of cell proliferation, cell specification, migration, and body axis patterning (Chou and Tole, 2019; Hunter and Rhodes, 2005; Singh et al., 2021; Yasuoka and Taira, 2021). Amongst the LIM-HD genes, Lhxl is known for its role in the development of the Mullerian tract (Huang et al., 2014), Lhx8 is required for oocyte development after the germ cells enter meiosis (Choi et al., 2008) and Lhx9 is essential for the development of bipotential gonads (Birk et al., 2000). We have also shown that multiple members of the LIM-HD gene family and their co-regulators are dynamically expressed in the developing mouse gonads specifically during the window of sex determination (Singh et al., 2021). However, the functional role of most LIM-HD family genes in gonad development and differentiation has not been explored.

In the present study, we investigated the expression profiles of LIM-HD genes in the gonads of multiple species and determined the role of one of the LIM-HD genes, Lhx2 in gonad development. Our results revealed that amongst the LIM-HD genes, expression of Lhx2 is sexually dimorphic with higher levels in the female gonads during sex determination in multiple species. We further observed that loss of Lhx2 in the XX mouse gonads leads to ectopic migration of the endothelial cells without causing somatic cell sex reversal. 


\section{Materials and Methods}

\section{Expression profiling of $L I M-H D$ genes in the developing gonads}

To investigate the expression pattern of LIM homeobox genes in developing gonads of different species, publicly available bulk RNA seq datasets of mice, rats and humans were analyzed (Cardoso-Moreira et al., 2019). To determine the expression profiles of Lhx2 in species with different modes of sex determination, we analyzed bulk RNAseq datasets of lizard (Whiteley et al., 2021), turtle (Czerwinski et al., 2016), chicken, and opossum (Cardoso-Moreira et al., 2019). Levels of Lhx2 in mice and humans were also analyzed in two additional datasets (Lecluze et al., 2020; Zhao et al., 2018). The accession numbers of these datasets are given in Supplementary Table 1.

\section{Determining the cell types that express $\operatorname{Lh} x 2$}

To determine the cell types in the mouse gonads that express $L h x 2$, we analyzed its mRNA expression single-cell RNAseq dataset (GSE128553) of mouse gonads at E13.5 (Ge et al., 2021). This dataset contains information on 4,842 cells from the $X X$ gonads at E13.5. We performed t-distributed Stochastic Neighbor Embedding (tSNE) and clustering on all the cells in the dataset to identify various cell types. After the tSNE projection, we used Kdr and Flt 1 as specific markers to identify the endothelial cell population, the germ cell population was visualized using the classical germ cell markers $D d x 4$ and Dazl. To visualize the expression of selected genes, we coloured the tSNE plots by imputed gene expression as computed by the MAGIC algorithm (van Dijk et al., 2018).

\section{Lhx2 knockout mice}

The study was approved by the institutional animal ethics committee (IAEC Project No., 27/14, 2/16). Heterozygous male and female mice with a targeted disruption of Lhx2 (Porter et al., 1997) and those with conditional Lhx2 allele (Mangale et al., 2008) were used in this study. The $L h x 2^{\text {flox/flox }}$ mice crossed with mice expressing Cre under tamoxifen responsive estrogen receptor promoter (Supplementary Fig 1) were used to conditionally delete the Lhx2 allele. All these strains were kindly provided by Prof. Shubha Tole, TIFR, Mumbai, India. The strains were bred at the experimental animal facility of ICMR-NIRRCH. Animals were mated in a 2:1 ratio and the day of vaginal plug observed was considered as embryonic day (E) 0.5 . 
The strategy of obtaining $L h x 2^{\text {flox/flox }}$ pups is shown in Supplementary Fig 1 . The $L h x 2^{\text {flox/flox }}$ pregnant females were fed orally with a single dose of $10 \mathrm{mg} / \mathrm{ml}$ of tamoxifen (Sigma Aldrich; MO, USA) micronized in corn oil (Sigma Aldrich) on E9.5. This dose and time were chosen as it is known to flox $L h x 2$ allele within 24h (Mangale et al., 2008). Preliminary experiments conducted in the gonadal tissue also showed adequate floxing and down regulation of Lhx2 mRNA at this dose and time (Supplementary Fig 1). As controls, $L h x 2^{\text {flox/flox }}$ mice without the tamoxifen-inducible CreER were fed orally with equivalent amounts of tamoxifen.

In the present study, mice with tamoxifen-inducible CreER and the Lhx2 flox alleles treated with tamoxifen are referred to as $L h x 2^{\text {flox/flox }}$ while those without the CreER and treated with tamoxifen are labelled as wild type (WT) controls. In the case of standard knockouts, the homozygous knockout pups are referred to as Lhx2-/- and the homozygous wild types as WT. Gonads were collected between E11.5 to E15.5 from the $L h x 2-/-$ or the $L h x 2^{\text {flox/flox }}$ strain.

\section{Allelic genotyping and embryonic sex genotyping}

To determine the Lhx2 genotype and the fetal sex, PCR was done using the Terra ${ }^{\mathrm{TM}}$ PCR Direct PCR mix (Takara Bio, Kusatsu, Shiga Japan). Genotyping for Lhx2 was performed as described earlier (Mangale et al., 2008). The PCR reactions with $L h x 2$ primer pairs give a 385 bp band for the WT allele and a $550 \mathrm{bp}$ band for the mutant allele. The sex of the embryos was determined using the primer pairs for the Jarid gene as described earlier (Clapcote and Roder, 2005). The primer sequences are given in Supplementary Table 2.

\section{RNA extraction, cDNA synthesis, and qPCR}

Gonads were dissected and the mesonephros was carefully separated. The gonads were stored in RNA later (ThermoFisher Scientific, MA, USA). RNA was extracted using the RNeasy mini kit (Qiagen, Germany) on column-based DNase I (ThermoFisher Scientific) digestion was performed following the manufacturer's protocol. cDNA was synthesized using High Capacity cDNA synthesis kit (Applied Biosystems, ThermoFisher Scientific, MA, USA) and quantitative PCR was done using Eva Green chemistry (BioRad Laboratories Inc., CA, USA) in the CFX96 Real-Time PCR System (BioRad) as detailed previously (Godbole and Modi, 2010; Mishra et al., 2020a). Each PCR was run for 35 cycles which involved initial denaturation of $95^{\circ} \mathrm{C}$ for $30 \mathrm{sec}$, primer annealing at optimized temperature for $30 \mathrm{sec}$, and extension at $72^{\circ} \mathrm{C}$ for $30 \mathrm{sec}$ with a final extension step at $72^{\circ} \mathrm{C}$ for $5 \mathrm{~min}$. PCR 
amplifications were carried out in duplicates, for all biological replicates. Primer efficiency value for each primer pair was calculated from standard curve analysis of serially diluted cDNA. The primer sequences, their annealing temperatures, and expected product sizes are given in Supplementary Table 2. Data were analyzed using the Pfaffl method as described earlier (Godbole and Modi, 2010).

\section{RNA Sequencing}

The strategy of gonad collection for RNAseq is shown in Supplementary fig 1. Pregnant females were fed with tamoxifen (as described above) and the pups were collected on E12.5. The gonads were separated from the mesonephros and preserved in RNA later (ThermoFisher Scientific). A minimum of 30 pairs of gonads each from the WT and $L h x 2^{\text {flox/flox }}$ embryos were pooled and outsourced for RNA sequencing (Sandor Biosciences, Hyderabad, India) using Illumina NextSeq 500 platform. Illumina TruSeq adapters were used; the read length was 76 nucleotides and was paired at both ends. An average of 45 million raw reads was generated per sample. Alignment was done using Tophat-2.0.13 using mm84 sequence as reference (ftp://ftp.ensembl.org/pub/release-84/fasta/mus_musculus/dna/).

\section{Differential gene expression analysis}

Fold change in FPKM values in $L h x 2^{\text {flox/flox }}$ ovaries was calculated with respect to WT ovaries. Genes with a fold change of 0.5 or less were taken as downregulated and fold change of $\geq 1.5$ is upregulated. The differentially expressed genes were used for Gene Set Enrichment Analysis (GSEA) using the WEB-based GEne SeT AnaLysis Toolkit (http://www.webgestalt.org/) at the default settings.

\section{Histology and Immunohistochemistry}

To study histology, mesonephros gonad complex was fixed in $4 \%$ paraformaldehyde (Sigma Aldrich) and 5um thick paraffin sections were stained with Hematoxylin-Eosin as detailed earlier (Mishra et al., 2020b). Immunofluorescence was performed as described previously (Godbole et al., 2017; Tiwari et al., 2021). In brief, sections were deparaffinized and epitopes were exposed in antigen retrieval buffer (Tris-EDTA pH-6.0). Blocking was done in $1 \%$ Bovine Serum Albumin (MP Biomedicals; Maharashtra, India) and the sections were probed overnight in 1:10 diluted anti-VE-Cadherin antibody (mouse monoclonal 8E5 clone, ELK Biotech, Wuhan China, catalogue \# EM1322). Negative controls were incubated either with 
isotype controls or Phosphate Buffered Saline (PBS) pH 7.2 instead of the primary antibody. The next day, slides were washed thrice in PBS, and detection was done using the Alexa 594 conjugated donkey anti-mouse antibody (Invitrogen, ThermoFisher Scientific, MA, USA Cat \# A-21203). The sections were visualized and images were captured using a fluorescence microscope equipped with a sCMOS camera (Leica Microsystems DMi8, Mannheim, Germany). The hematoxylin-eosin stained sections were viewed under a bright-field microscope (Olympus; Tokyo, Japan) and representative areas were imaged using a digital camera (Olympus). From the images, the desired areas were extracted using ImageJ and digitally enhanced. All quantifications were done before image enhancements.

\section{In Vitro Gonad Recombination Assay and estimation of branching pattern}

The scheme of the gonad recombination assay is described in Supplementary Fig 2. Breeding pairs of FVB mice constitutively expressing Green fluorescent protein (GFP) were originally obtained from the National Institute of Immunology (NII), New Delhi, India. Mice were bred and maintained at the Experimental Animal Facility of ICMR-NIRRCH. For gonad recombination assays, the pups from FVB-GFP and Lhx2 heterozygous mothers were collected on E11.5. The GFP mesonephros and the gonads were recombined in agarose blocks. The dimensions of agar mould and the protocol are detailed elsewhere (Capel and Batchvarov, 2008). Briefly, the sterile moulds were poured with sterile $1.5 \%$ agar (prepared in DMEM-F12) and allowed to cool. The agar blocks were removed and equilibrated in DMEM-F12 with $10 \%$ fetal bovine serum and antibiotic-antimycotic mixture (all from Gibco, ThermoFisher Scientific, MA, USA). The gonads and mesonephros were carefully dissected and the GFP mesonephros (of either sex) was carefully overlayed with the gonad from WT or Lhx2-/- ovaries in the ridges of the agar mould. XY gonads from WT pups were used as positive controls. The gonadal assembly was cultured at $37^{\circ} \mathrm{C}$ with $5 \% \mathrm{CO}_{2}$ and migration of the GFP positive cells was monitored at 24, 48, and 72 hours post culture. Recombinants were imaged on a stereomicroscope equipped with fluorescent imaging (Olympus).

\section{Quantification of angiogenesis}

For quantification of angiogenesis patterns, the angiogenesis analyser macros in ImageJ software (https://imagej.nih.gov/ij/macros/toolsets/Angiogenesis Analyzer.txt) was used (Carpentier et al., 2020). The desired areas were selected, the background was corrected and 
the images were subjected to the angiogenesis analyser that quantified various branching parameters. The details of the parameters and their interpretation are provided in (Supplementary Table 3). All the images of the recombinant gonads were blinded for their sex and genotype and then analyzed. Data from 3 independent experiments ( $n=3$ gonads) each from WT XX, WT XY, and Lhx2-/- XX at 72h of recombination was analyzed. Mean and standard deviation was computed and statistical analysis was applied.

\section{Assessment of endothelial cell transcriptome}

We used the previously curated dataset of cell-type-specific transcriptome where the genes enriched in the endothelial cells of both the sexes are enlisted (Jameson et al., 2012). This data set is generated out of microarray analysis of flow-sorted Flkl-mCherry positive endothelial cells from $\mathrm{XX}$ and $\mathrm{XY}$ mouse gonads at E11.5, E12.5, and E13.5. The dataset contains 473 genes at E12.5 whose expression is significantly higher in endothelial cells as compared to other cell types ( $\mathrm{p}$-value cutoff of 0.05 , and a fold change cutoff of 1.5). Information for 445/473 endothelial cell-enriched genes was available in our RNAseq dataset. Their FPKM levels were compared between WT XX vs WT XY vs $L h x 2^{\text {flox/flox }}$ XX gonads at E12.5. Those genes whose expression in the $\mathrm{XY}$ gonads was higher than those of $\mathrm{XX}$ were considered male-biased and those with expression lower in XY gonads as compared to $\mathrm{XX}$ were considered female-biased (fold change $>1.25$ or $<0.5$ respectively).

\section{Data analysis and presentation}

All the data were analyzed statistically using ANOVA and graphs were plotted using GraphPad Prism (Version 8, California). Heatmaps were plotted using Morpheus (https://software.broadinstitute.org/morpheus). The GSEA results were visualized using the R studio version 3.6.2 with ggplot2 packages (RStudio Team, Boston, MA http://www.rstudio.com/) as described previously (Ashary et al., 2020; Colaco et al., 2021). The graphical abstract and experimental schematic in supplymentery figures were created with BioRender.com 


\section{Results:}

\section{Expression profiles of $L I M-H D$ genes in developing mouse, rat, and human gonads}

Fig 1A shows expression profiles of twelve $L I M-H D$ genes that are present in the mammalian genome. As evident, Lhx5 is not expressed in the mouse, rat, and human gonads at the time of sex determination. The others are expressed at varying levels with some being sexually dimorphic while others are equally expressed in both the sexes. In general, most LIM-HD genes were expressed in a female dominant manner in the mouse and human gonads, while in rat gonads some of them were male dominant. For example, Lmxlb is female dominant in $\mathrm{XX}$ mouse and human gonads while it is XY dominant in rat gonads. Some genes were sexually dimorphic only in one species but not others. For example, Isll and Isl2 are largely female dominant in the human gonads but not in the mouse and rat gonads; Lhx4 is female dominant only in the rat gonads. Intriguing, irrespective of the species, Lhx2 was XX dominant during sex determination in all three species. In the mouse, as early as E10.5 Lhx2 was XX dominant and remained high throughout the window of sex determination. In the rat, Lhx2 became sexually dimorphic slightly later (just after the onset of sex determination, E15), while in the humans, it was XX dominant right from 7 weeks and remained high in the female gonads throughout (until 11 weeks).

We performed RT-PCR for Lhx2 and Lhx9 in the XX and XY mouse gonads at E12.5 and E13.5. As expected, both these genes were expressed in XX and XY gonads (Fig 1B). Quantitative analysis showed that at both E12.5 \& E13.5, Lhx2 was significantly higher in the $\mathrm{XX}$ as compared to $\mathrm{XY}$ mouse gonads (Fig 1C). As predicted from the RNAseq data, Lhx9 was not sexually dimorphic at E12.5 and E13.5 (Fig 1C). 
A

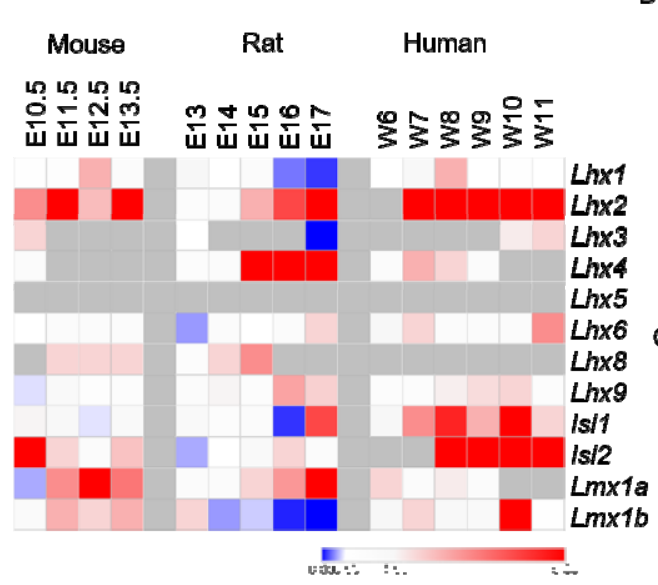

B

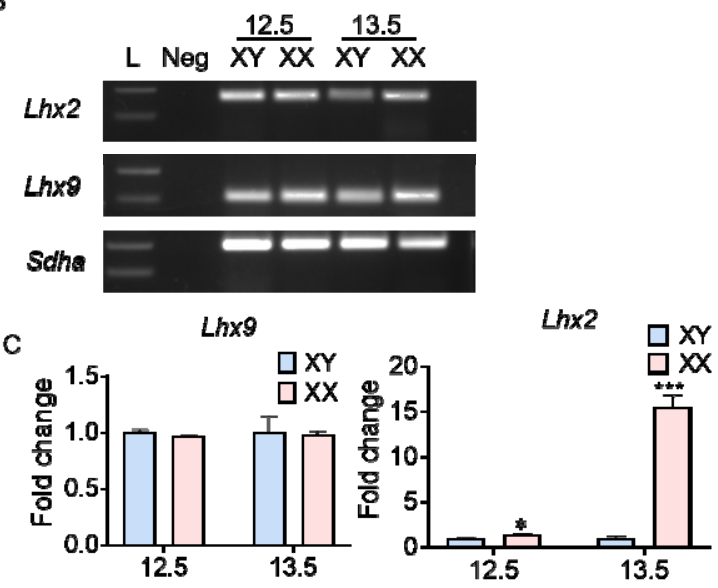

Fig 1: Expression profile of LIM homeobox genes in mammalian developing gonads. (A) Heatmap demonstrating the ratio of FPKM values (XX/XY) for LIM homeobox genes (Lhxi-6, Lhx8-9, Is $11-2$, Lmxla-1b) in gonads. Each row is the gene and each column is the time point in embryonic (E) development in days for the mouse and rat and weeks $(W)$ in humans. FPK.M values were extracted from RVA-seq dataset (Cardoso et al.,2019). Boxed in red shades indicate female dominance while those in blue shades indicate male dominance. White boxes indicate the levels are not sexually dimorphic (XX/XY ratio $>0.5$ or $<1.5$ ). Grey are missing values. (B) Gel image showing expression of $L h x 2$ and $L h x 9$ by RT-PCR in developing gonads at E12.5 and E13.5. Stha is housekeeping gene and L is molecular marker of $100 \mathrm{bp}$. Neg lane is loaded with no DNA template control (C) Quantification of Lhx2 and Lhxs mRNA levels by QPCR in XX and XY gonads at E12.5 and E13.5 (X axis). Values were normalized to Sdha and the data is expressed in fold change of the mean value obtained for $X Y$ gonads. Values on $Y$ axis are mean $+S D(n=3$ biological replicates/time point). $p<0.05$, 部南 $p<0.001$

\section{Sexual dimorphic expression of $L h x 2$ is evolutionarily conserved}

Since Lhx2 was sexually dimorphic with higher expression in $\mathrm{XX}$ when compare to $\mathrm{XY}$ gonads in mice, rats, and humans, we sought to compare its expression in the gonads of other species with environmental or genetic (XX/XY and ZW/ZZ) modes of sex determination during gonad development. The red-eared slider turtle (Trachemys scripta elegans) has a temperature-dependent sex determination mechanism (Czerwinski et al., 2016). In this species, eggs incubated at female promoting temperature (FPT, $31{ }^{\circ} \mathrm{C}$ ) had higher expression of $L h \times 2$ as compared to those incubated at male promoting temperature (MPT, $26^{\circ} \mathrm{C}$ ) from the onset of sex determination. One of the species of lizards (Pogona vitticeps) uses the genetic and temperature-dependent sex determination mechanisms, and higher temperature $>32^{\circ} \mathrm{C}$ can override the genetic mode of sex determination (Whiteley et al., 2021). In this species, throughout sex determination, Lhx2 was 3-6 folds higher in the eggs incubated at FPT as compared to MPT irrespective of their genetic sex (Fig 2). Chicken has ZW/ZZ mechanism of sex determination and the female is heterogametic (Capel, 2017). In the chicken (Gallus gallus), Lhx2 expression was 2-4 folds higher in the gonads of ZW (female) 
embryos as compared to ZZ (male) during the period of sex determination (Fig 2). Opossum (Monodelphis domestica) has XX/XY mechanism of sex determination and the SRY gene has first evolved (Katsura et al., 2018). In this species too, irrespective of the time point, $\mathrm{XX}$ gonads had 7-20 folds higher expression of $L h x 2$ as compared to XY gonads (Fig 2). In mice and humans, both of which utilize the XX/XY mode of sex determination, Lhx2 was female dominant in both species. In mice, Lhx2 was at least 2-4 folds higher in XX gonads as compared to XY. However, in humans, $L H X 2$ expression was $15-18$ folds higher in the $\mathrm{XX}$ gonads between W9-12.
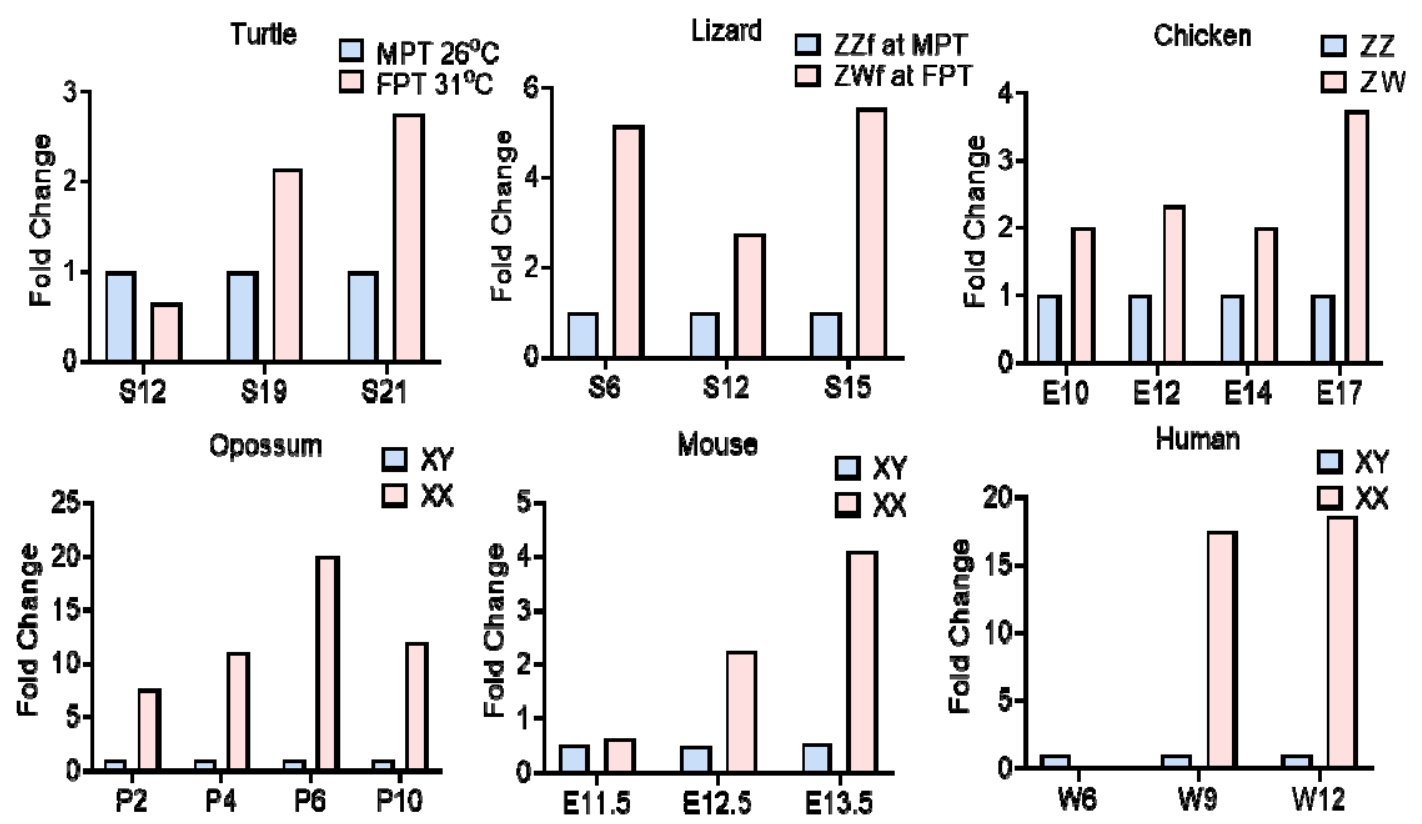

Fig 2: Sexually dimorphic expression of $L h x 2$ in developlng gonads of specles with different mechanisms of sex determination. Bulk RNA seq datasets of turtle (SRP079664; Czerwinski et al., 2016), lizard (PRJNA699086; Whiteley et al., 2021), chicken (E-MTAB-6769; Cardoso-Moreira et al., 2019), opossum (E-MTAB-6833; Cardoso-Moreira et al., 2019), mouse (SRP076584; Zhao et al., 2018) and human (GSE116278; Lecluze et al., 2020) were extracted and analysed. Expression values in each species were converted to fold change with respect to $X Y$ values at one and plotted on $Y$ axis. $X$ axis shows the time point of embryonic development.

\section{Lhx2 is dispensable for gonad development and somatic cell sex determination}

Gonads from the Lhx2-/- embryos were well developed with distinct mesonephros and gonad proper. Histologically, Lhx2-/- XX gonads were indistinguishable from that of the WT controls at both E13.5 and E15.5 (Fig 3A). Although in some instances, the XX gonads of Lhx2-/- embryos at E15.5 appeared rounded and attached to the mesonephros (data not 
shown). There was no evidence of tubular organization in the $L h x 2-/-\mathrm{XX}$ gonads like that seen for the XY gonads at E13.5 and E15.5 (Fig 3A)

We analyzed the levels of key genes involved in sex determination in the developing $L h x 2^{\text {flox/flox }}$ gonads at E12.5 and observed that most of these genes (except Sf1) were expressed similarly at identical levels in $L h x 2^{\text {flox/flox }}$ gonads as compared to their WT counterparts (Supplementary Fig 1). We also analyzed the mRNA levels of male somatic cell-specific genes Dmrt1 and Sox9 at E13.5 and E15.5 by qRT-PCR. As expected, both these genes were sexually dimorphic with higher expression in $\mathrm{XY}$ gonads as compared to $\mathrm{XX}$ gonads at E13.5 and E15.5. The levels of these genes did not significantly differ in ovaries of WT and Lhx2-/- embryos (Fig 3B and 3C respectively). As expected, the mRNA level of Foxl2 (female somatic cell marker) was higher in the WT XX gonads as compared to the WT XY gonads. The level of Foxl2 was identical in the WT and Lhx2-/- XX gonads at E13.5 and E15.5 (Fig 3B and 3C respectively).
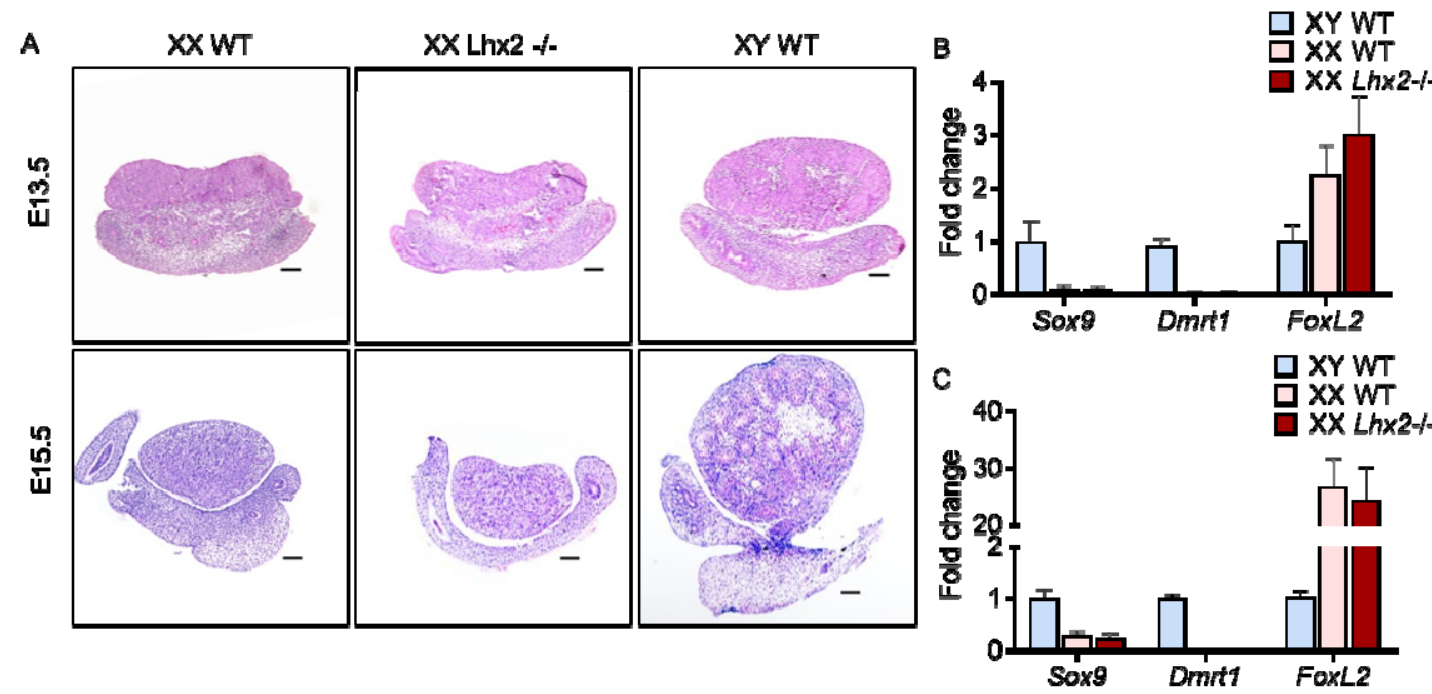

Fig 3: Somatic sex is not reversed in absence of $L h \times 2$. (A) Histology of wild type (WT) $X Y, X X$ and

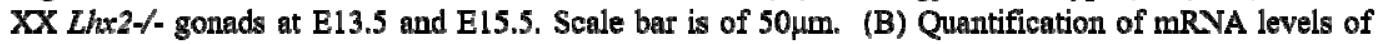
Sox9 and Dmrtl (Sertoli cell marker) and For/2 (Granulosa cell marker) by qPCR in the WT XY, XX and $L h x 2 \%-\mathrm{XX}$ gonads at E13.5. (C) Quantification of mRNA levels of Sox9 and Dmrtl (Sertoli cell marker) and Foxi2 (Granulosa cell marker) by qPCR in WT XY, XX and Lhx2- XX gonads at E15.5. Values for Sdha were used for normalization. Data is presented as a mean+SD of fold change of the WT $\mathrm{XY}$ gonads for 6 biological replicates/time point/sex/genotype.

\section{Identification of genes regulated by $L h x 2$ in the XX gonads}

To address the roles of $L h x 2$ in the developing XX gonads, we carried out RNA seq of WT XX, WT XY, and Lhx $2^{\text {flox/flox }} \mathrm{XX}$ gonads at E12.5. A total of 4076 genes were found to be 
differentially expressed in the $L h x 2^{\text {flox/flox }} \mathrm{XX}$ gonads as compared to WT. Amongst these, 2566 genes were upregulated while 1510 were downregulated in the $L h x 2^{\text {flox/flox }}$ ovaries as compared to the wild type ovaries (Supplementary Table 4). Of the down-regulated genes, 33 genes were exclusively expressed in the WT XX gonads but not in the $L h x 2^{\text {flox/flox }} \mathrm{XX}$ gonads (Supplementary Table 4). Isg15 was the most upregulated gene, while Lgals3 was the most down-regulated gene in the $L h x 2^{\text {flox/flox }}$ ovaries (Supplementary Table 4).

Differentially expressed genes were analyzed using GSEA (Fig 4). The results revealed that biological processes like organ or tissue immune responses, protein-DNA complex subunit organization, and hepatobiliary development were overrepresented, while synaptic transmission, sensory perception to taste, cilium development were under-represented (Fig 4A). Amongst the molecular functions, semaphorin receptor binding, double-stranded RNA binding, and beta-catenin binding were overrepresented while peptidase activity, structural constituents of the ribosome, electron transfer activity, and antioxidant activity were significantly underrepresented (Fig 4B). The panther pathways associated with vascular functions such as angiogenesis, PDGF and WNT signalling pathway, insulin/IGF1 protein kinase B signalling cascade, and cadherin signalling pathways were significantly overrepresented (Fig 4C). The pathway associated with DNA replication were underrepresented (Fig 4C). The pathway enrichment plots and expression profiles of a subset of genes contributing significantly to the enrichment of angiogenesis and PDGF signalling pathways are shown in Fig 4D. The enrichment plots of other selected biological processes and pathways are shown in Supplementary Fig 3. 


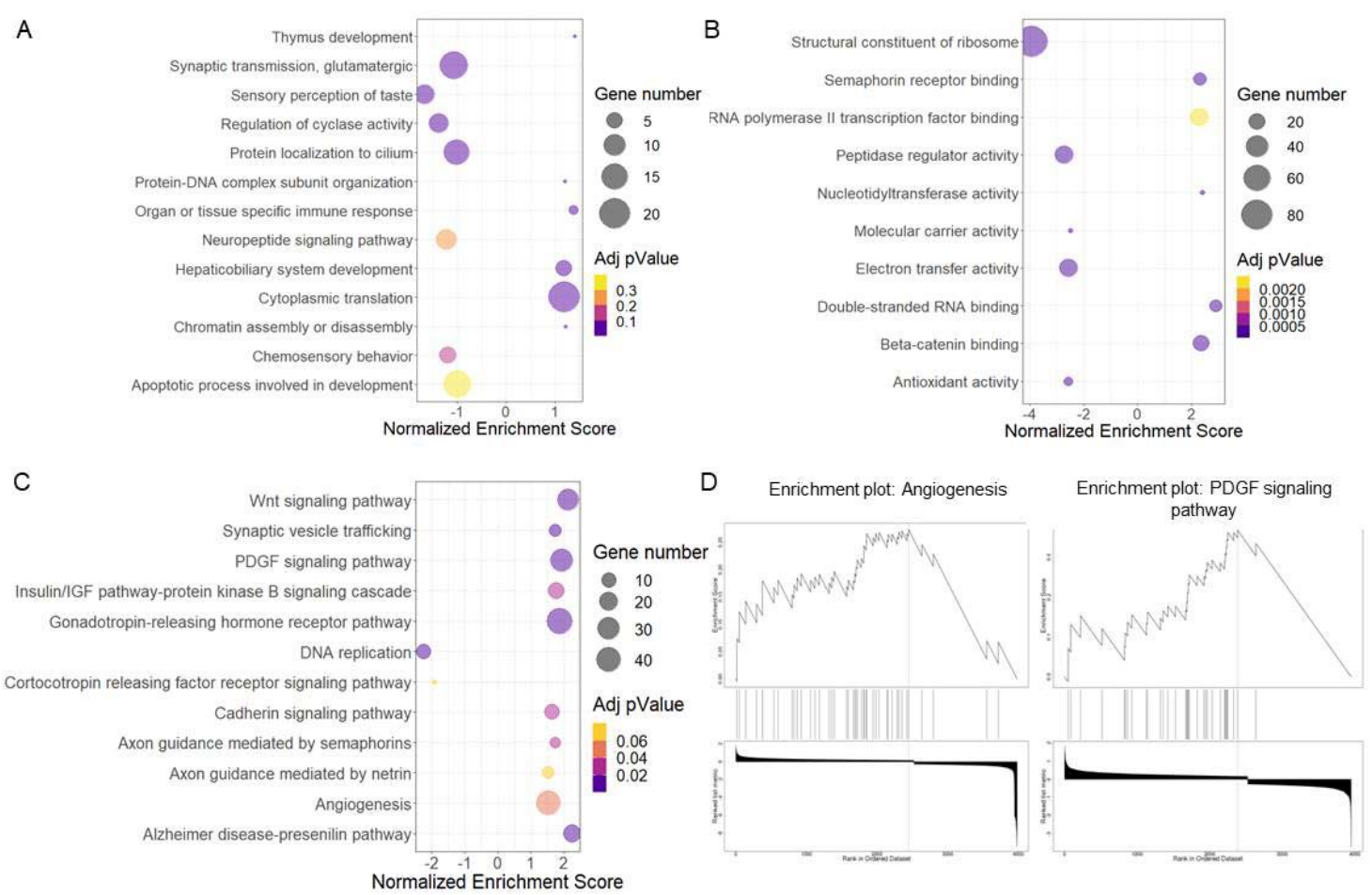

Fig 4: Gene Set Enrichment Analysis (GSEA) for the differentially expressed genes in Lhx2-/- XX gonads. The differentially expressed genes between wild type and $L h x 2^{\text {flox flox }} \mathrm{XX}$ gonads at E12.5 were subjected to GSEA using the WEB-based GEne SeT AnaLysis Toolkit (http://www.webgestalt.org/). (A) The enriched biological processes, (B) molecular Functions and (C) panther pathways are shown as bubble plots. $\mathrm{X}$ axis is normalized enrichment score where the highly enriched processes are skewed on the right. The numbers of genes and the adjusted $\mathrm{p}$ values for each process is shown. (D) Enrichment plots for the angiogenesis pathway and PDGF signaling pathway. Y axis represents enrichment score which shows the degree of overrepresentation of gene sets and vertical bars shows where the member of the gene set appeared in ranked list. The bottom part of each graph shows ranking matrix that measures the gene's correlation with their phenotype.

We performed immunofluorescence for VE-Cadherin and observed a higher number of endothelial cells in the WT XY gonads as compared to XX WT gonads (Fig 5C). However, many VE-Cadherin-positive cells were detected in the ovarian stroma of E13.5 in the Lhx2-/XX gonads (Fig 5C). The negative control sections incubated with an isotype IgG did not show any reactivity (Fig $5 \mathrm{C}$ inset). 
A Up regulated

B $\quad$ 口XXWT

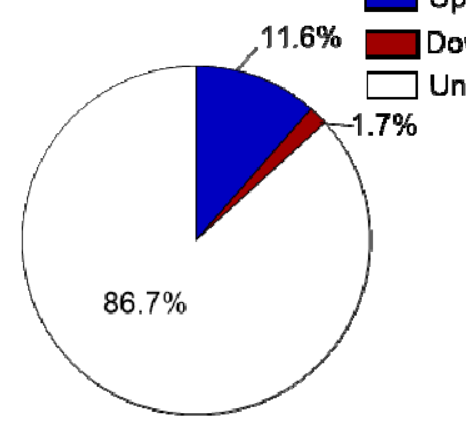

Down regulated 0.8 Unchanged

C

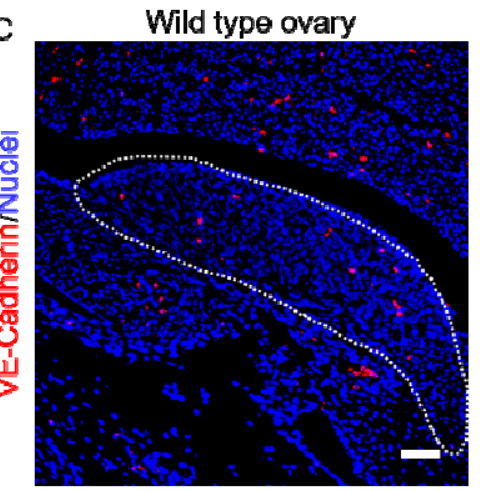

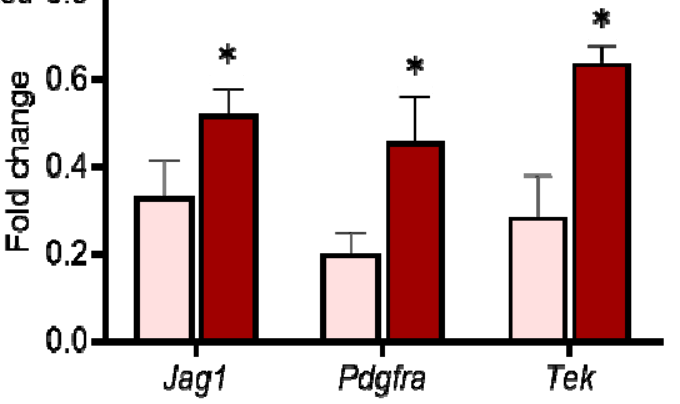

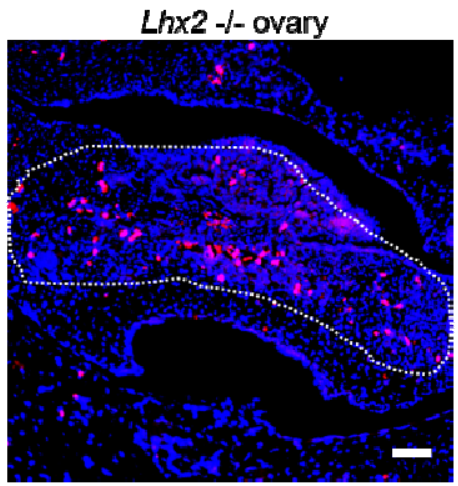

Wild type testis

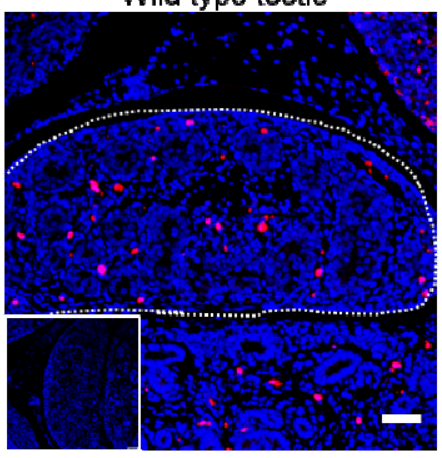

Fig 5: Endothelial genes are enriched in the ovaries lacking $L h x 2$. (A) Percentage of endothelial cell specific genes altered in the ovaries of $L h x 2^{\text {flox }}$ illox embryos as compared to wild type (WT) XX at E12.5. (B) qPCR for endothelial enriched genes at E13.5 in WT and $L h x 2-/-$ $\mathrm{XX}$ gonads. Data is presented as fold change where mean values of was taken as 1 . Values are mean+SD of three biological replicates. ${ }^{* *} p<0.01$, *** $p<0.001$ (C) Immunofluorescence for VE-Cadherin (red channel) in wild type wild type and $L h \times 2-/-$ ovaries and wild type testis at E13.5. Nuclei are stained with DAPI (blue channel). Negative control is shown in inset. Scale bar is $50 \mu \mathrm{m}$. The dotted area marks the gonad.

\section{Lhx2 is expressed in the germ cells and not in endothelial cells of XX gonads at E13.5}

After filtering for low-quality cells (cells with less than 200 genes and those expressed in less than 3 cells were removed). we obtained a total of 4,842 cells at E13.5. After performing tSNE, based on gene expression diversity we could identify a total of 17 independent clusters (Fig 6A). Of these Lhx2 was detected in the cells in three clusters (Fig 6B). However, none of these clusters expressed endothelium-specific genes $K d r$ and Flt1. Instead, the endothelium-specific genes were detected in a separate cluster of the tSNE plot (Fig 6B). The cluster of cells that expressed $L h x 2$, expressed the germ cell-specific marker $D d x 4$ (Fig 6B) and Dazl (Fig 6F) confirming that Lhx2 is expressed by the germ cells. Other cell types (if any) barely expressed $L h x 2$ and these were not endothelial cells (not shown). 
A
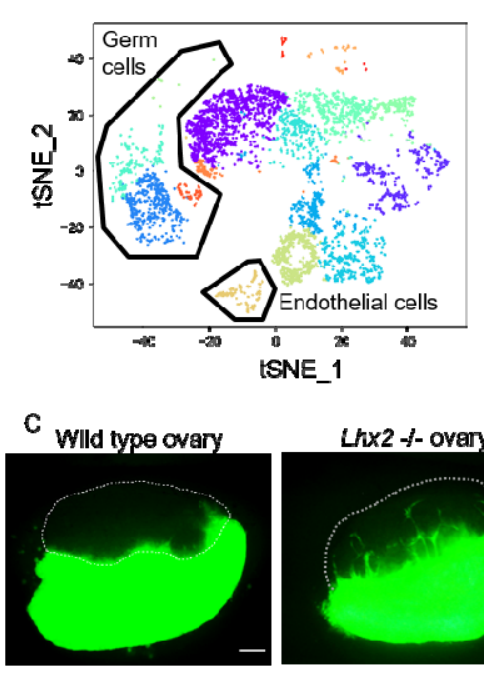

D

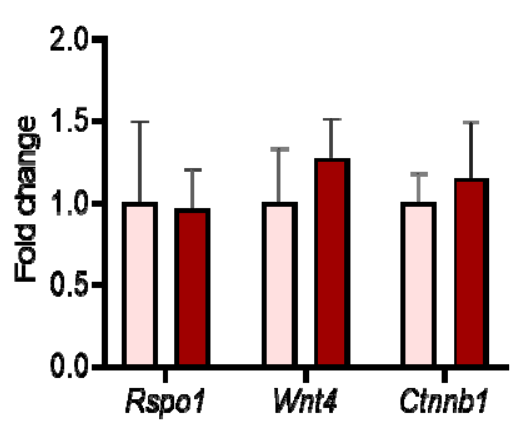

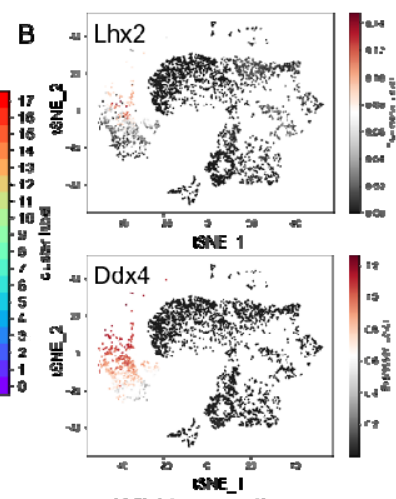

Lhx2 - ovary
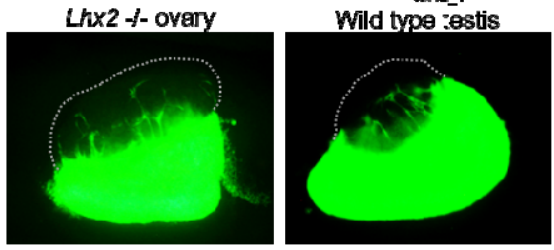

$X X W T$

$X X L h \times 2-1-$

$\mathrm{E}$
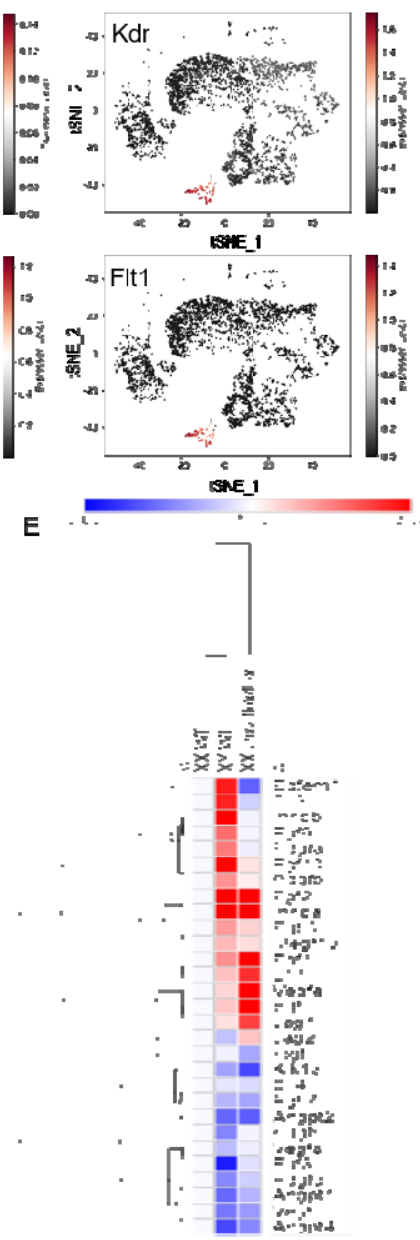

$\mathbf{F}$
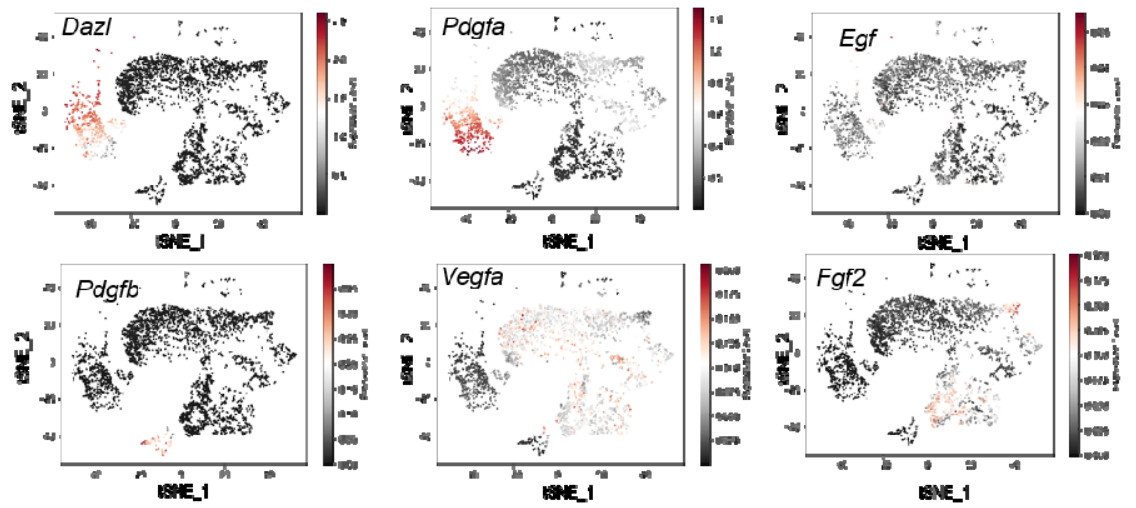

Fig 6: Lhx2 is expressed in the germ cells and its loss leads to excessive mesonephric endothelial coll migration in a coll non-autonomous manner. (A) tSNE plot of single cell RNA seq dataser (GSE128553) of mouse ovaries at E13.5 showing clustering of different cell types. (B) Imputed expression of $L / x \times 2$ and marker genes in the tSNE plot. The germ cell marker is Ddx 4 and endothelial cell markers are $K d$ and $F h$. (C) Gonad recombination assay where mesonephros from wild type (WT) $\mathrm{XX}$ or XY animals constitutively expressing GFP were overlaid with gonads from WT XY, WT XX and $\mathrm{XX} L h \times 2 \%$ - embryos. The co-cultures were monitored for mesonephric cell migration ar $48 \mathrm{~h}$. The region of the gonad is outlined. The scale bar is $100 \mu \mathrm{m}$ (D) qPCR of Rypol, Whit and Cimb1 i.2 $L / \mathrm{k} 2$ /- XX gonads at E13.5. Data is presented as fold change where mean values of WT was caken as 1. Values are mean+SD of three biological replicates. (D) Heatmap of secretory factors izvolved in encothelial cell migration in the WT XX, WT XY and $L h x 2^{\text {flowiter }} \mathrm{XX}$ gonads at E12.5. The relative scale is shown. (E) Imputed expression of genes involved in endothelial cell migration projected on the tSNE plot shown in A. $D a=L$ is a germ cell marker. 


\section{Lhx2 leads to excessive endothelial cell migration from the mesonephros in a cell non- autonomous manner}

Since $L h x 2$ is expressed by the germ cells and not the endothelial cells, we hypothesized that the large numbers of endothelial cells in the Lhx2-/- XX gonads could be due to their excessive migration in a cell non-autonomous manner. To test, if the angiogenesis observed in the Lhx2-/ XX gonads is cell-autonomously regulated, we performed a gonad recombination assay. As expected, irrespective of the genetic sex (XX/XY) of the mesonephros, by 48 hrs of incubation, GFP-positive cells were seen streaming into the XY gonads, but not in the XX gonads (Fig 6C). In the experiments where Lhx2-/- XX gonads were co-cultured with GFP expressing mesonephros, several GFP-positive cells could be detected in the gonads at various regions (Fig 6C). In the WT XX gonads, GFP-positive cells could not be detected even at $72 \mathrm{~h}$ of co-incubation (not shown) indicating the specificity of the assays.

Previous studies have shown that mice knockout for Wnt4, Rspol, or Ctnnbl have ectopic blood vessel development in XX gonads (Chassot et al., 2014). To test if the elevated endothelial cells migration in the Lhx2-/- XX gonads is due to loss of Wnt4, Rspol, or Ctnnb1, we performed qPCR for these three genes in XX gonads of WT and Lhx2-/- embryos at E13.5 (Fig 6D). The results revealed that the levels of Wnt4, Rspol, or Ctnnb1 were comparable in both groups. We also analyzed the level of $L h x 2$ from the RNAseq dataset of Wnt4 KO (Naillat et al., 2015). The results revealed that the mRNA levels of Lhx2 are identical in Wnt4 KO and WT embryos (Supplementary Fig 3F).

We next studied the mRNA levels of 29 secretory factors that are known to aid in endothelial cell migration and angiogenesis in WT XY gonads. As evident from Fig.6E, the mRNA levels of most of the angiogenic factors involved in endothelial cell migration are elevated in WT XY gonads and were also elevated in $L h x 2^{\text {flox/flox }}$ ovaries and those that are downregulated in WT XY gonads were also down-regulated in the $L h x 2^{\text {flox/flox }}$ ovaries at E12.5 (Fig 6E).

To test if any of these factors are secreted by the germ cells we analyzed their expression in the single-cell RNAseq dataset. The tSNE plots (Fig 6F) of the selected genes revealed that $P d g f a$ is abundantly transcribed by most germ cells, a small subset of germ cells also 
transcribed Egf. The others were expressed by the supporting cells including the endothelial cells (Fig 6F).

\section{Male-like behavior of the endothelial cells in the XX gonads in absence of $L h x 2$}

To characterize the endothelial cells in the XX gonads in absence of $L h x 2$, we analyzed the expression profiles of 445 genes that are known to be enriched in the endothelial cells (Supplementary Table 5). The results revealed that 107/445 genes were male-biased $(>1.25$ folds in the XY gonads with respect to XX gonads) while 52/445 genes were female-biased $(<0.5$ folds in the $\mathrm{XY}$ gonads with respect to $\mathrm{XX}$ gonads) at E12.5. Interestingly, the expression of $62 / 107(\sim 58 \%)$ male-biased endothelial cells was elevated in the $L h x 2^{\text {flox/flox }}$ ovaries (Fig 7A, Supplementary Table 5).

To test if the endothelial cells in the Lhx2-/- XX gonads are capable of organizing into a network like that in the testis, we compared the branching patterns of the GFP positive cells in the gonad recombination assay of WT XY and $L h x 2-/-\mathrm{XX}$ gonads at $72 \mathrm{~h}$ of co-culture. As expected, WT testis had distinct master segments, branches, and twigs with an intricate meshwork (Fig 7B). Almost a similar meshwork was also seen in Lhx2-/- ovaries (Fig 7B). Supplementary Fig 4 gives the representative images for the nodes, extremities, and mesh pattern in the WT testis and Lhx2-/- ovaries.

We quantified the pattern of branching in both the conditions ( $\mathrm{n}=3$ each) and observed that the numbers of extremes, nodes, junctions, twigs, and branches were significantly higher in the Lhx2-/- ovaries as compared to WT testis (Fig 7C). However, the number of segments, master segments, isolated segments, and master junctions were comparable in WT testis and Lhx2-/- ovaries (Fig 7C). The mesh area was significantly lower in Lhx2-/- XX gonads as compared to WT testis (Fig 7C).

We next asked if the endothelial cells that migrated in the Lhx2-/- ovaries form a coelomic blood vessel (Fig 7D). In WT testis (n-10) at E13.5, a distinct coelomic blood vessel was detected which was not observed in WT XX gonads (Fig 7D). Although some vascularization was observed in Lhx2-/- XX gonads ( $\mathrm{n}=10)$, there was no distinct coelomic blood vessel akin to WT testis. We also analyzed the gonads at E15.5 where a distinct vascular network was seen in the XY gonads $(n=10)$ but this was not evident in the XX gonads (Fig 7D). In most Lhx2-/- XX embryos, the gonads $(\mathrm{n}=15)$ had hemorrhagic areas, a distinct coelomic blood vessel was not observed (Fig 7D). 

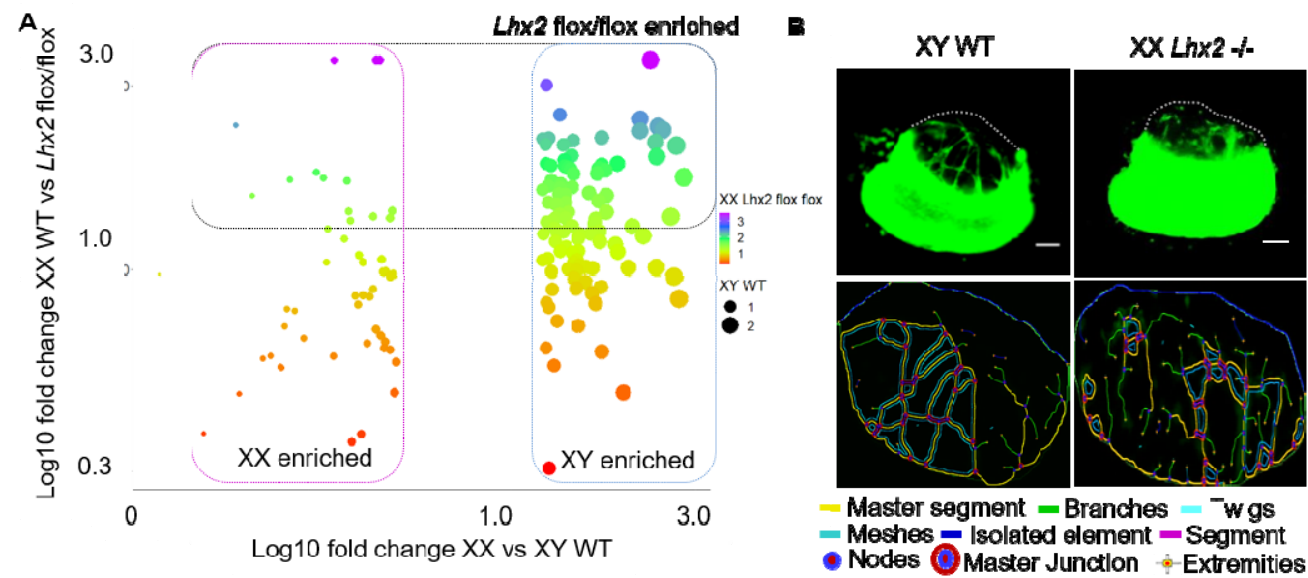

C

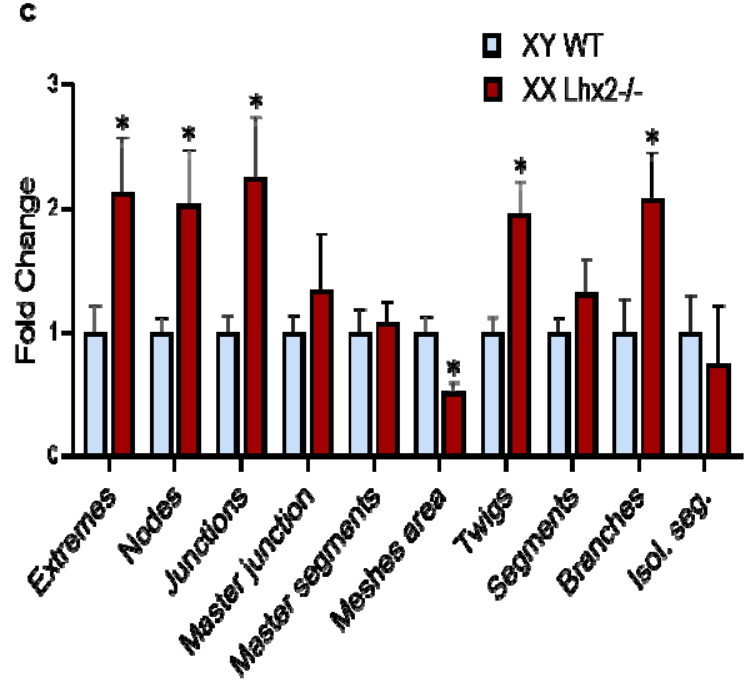

D

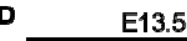

E13.5

E15.5

Fig 7: Masculintzation of the ondothelial cells in the XX gonads in absence of $L h x 2$. (A) Bubble plul showing expression levels of endolhelial enriched genes (al E12.5) in wild lype (WT) XY and in XX $L h \times 2^{\text {tes/10x }}$ gonads normalized to the values observed in WT XX gonads. (B) Gonad recombination assay at $72 \mathrm{~h}$ of co-culture. XY WT, and XX $L / 42-/-$ gonads were used for gonadal recombination assay and observed after $72 \mathrm{~h}$ of co-culture. Recombinant gonads were analyzed using Imagel software with angiogenesis analyzer plugin. The different patterns of branching are shown. Scale bar is of $100 \mu \mathrm{m}$ (C) Graph were plotted using fold change of different parameters of angiogenesis. Values are mean-SD for 3 biological replicates and indicates $\mathrm{p}<0.05$. (D) Brightfield images of XX WT. XY WT and XX

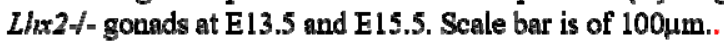

\section{Discussion}

In the present study, we demonstrate that multiple LIM-HD genes are expressed in the developing gonads during the window of sex determination. Amongst these, Lhx2 is predominantly expressed in the developing ovaries in most species irrespective of the mode of sex determination. In the mouse ovaries, Lhx2 is exclusively expressed in the germ cells and its loss leads to an increase in endothelial cell migration in a cell-non autonomous manner. 
LIM-HD genes are transcription factors that play a fundamental role in cell fate determination and patterning of somatic tissues. In the context of gonad development, three LIM-HD genes are known to play crucial roles. Lhxl has a role in Mullerian duct development; Lhx9 is required for the development of bipotential gonad and $L h x 8$ is essential for oocyte survival in the developing ovaries (Singh et al., 2021). However, along with these three, other members of the LIM-HD gene family are also dynamically expressed in the developing mouse gonads with many having a sexually dimorphic expression during the window of sex determination (Singh et al., 2021). Extending these findings, herein we show that except for Lhx5, mRNA for most members of LIM-HD genes are detected in the mouse, rat, and human gonads during the window of sex determination. Interestingly, many of these, have female biased expression during sex determination. These include Lhx2, Lhx4, Ils2, Lmxla, and Lmxlb; all of which are female biased in human gonads and also in one of the two rodent species. These results imply that LIM-HD genes might have a role in ovarian development. Indeed, LIM-HD binding elements are enriched in several meiosis-specific genes in the germ cells of developing ovaries (Li et al., 2013).

In the vertebrate gonads, sex-determining mechanisms are highly divergent where environmental cues and genes, individually or in combination operate to determine the fate of the bipotential gonads (Bhattacharya and Modi, 2021; Capel, 2017). Our analysis revealed that in the mouse, rat, and humans which have a genetic (XX/XY) mode of sex determination, $L h x 2$ is expressed in a sexually dimorphic manner with higher expression in females as compared to male gonads. To determine if the sexually dimorphic expression of Lhx2 is influenced by the mode of sex determination, we compared its mRNA levels in developing gonads of species with environmental (mainly temperature) and genetic (ZZ/ZW or $\mathrm{XX} / \mathrm{XY}$ ) mode of sex determination. The red-eared slider turtle has a temperaturedependent sex determination mechanism (Czerwinski et al., 2016) while Pogona vitticeps (a species of lizard) has both genetic and a temperature-dependent sex determination mechanism where temperature can override the genetic mode of sex determination (Whiteley et al., 2021). In both these species, levels of $L h x 2$ were higher in the developing ovaries as compared to testis. In the chicken which has exclusively ZW/ZZ mechanism of sex determination (Capel, 2017; Chue and Smith, 2011), Lhx2 mRNA was female dominant. The opossum is a marsupial (metatherian) species where the SRY gene has first evolved and has become operative during evolution (Capel, 2017; Katsura et al., 2018). In this species too, Lhx2 was over abundant in the female gonads as compared to males during the period of sex 
determination. Thus, our analysis indicates that female dominating expression of $L h x 2$ in the gonads is evolutionarily conserved and independent of the different mechanisms of sex determination. A very limited set of genes are known to be expressed in a sexually dimorphic manner in an evolutionarily conserved fashion during the period of sex determination and gonad differentiation (Bhattacharya and Modi, 2021; Capel, 2017). Our study adds Lhx2 as another gene whose expression is evolutionarily female biased. Based on its conserved expression profiles in the gonads across species, we hypothesized that $L h x 2$ might have a key role in ovarian development.

There are two mammalian orthologs of the drosophila apterous gene, $L h x 2$, and $L h x 9$, both of which have high sequence homology and have overlapping patterns of expression in the developing nervous system (Bulchand et al., 2003; Chou and Tole, 2019). Lhx9 has an indispensable role in gonad development as mice lacking $\operatorname{Lh} x 9$ do develop the genital ridges, but the somatic cells fail to proliferate and the gonad is arrested at the bipotential stage (Birk et al., 2000). However unlike $\operatorname{Lh} x 9$, Lhx2 appears to be dispensable for gonad development as in Lhx2-/- embryos, the gonads developed and differentiated correctly into ovaries. At E13.5 and E15.5, the ovaries were histologically indistinguishable from the WT counterparts. Together this data indicated that, unlike $\operatorname{Lh} x 9$ which is essential at the early steps of genital ridges, $L h \times 2$ is not involved in bipotential gonad development.

Sex determination in the developing gonads involves the differentiation of somatic cells into either Sertoli cells or pre-granulosa cells. A plethora of molecular players in a mutually opposing manner regulates the fate of somatic cells in the developing gonads. There are promale factors that suppress the expression of pro-female genes allowing Sertoli cell specification; in parallel, there are pro-female factors that suppress the expression of protestis factors allowing the specification of the pre-granulosa cells (Capel, 2017; Ortega et al., 2019; Rotgers et al., 2018; Ungewitter and Yao, 2013; Yildirim et al., 2020). Since the expression of Lhx2 was female biased, we hypothesized that it might have a role in female sex determination. However, contrary to our expectation, at E12.5, E13.5 and E15.5, Lhx2 knockout ovaries appropriately expressed the pre-granulosa cell markers, Foxl2, Wnt4, Rspol, and Ctnnb1. Also, there was no evidence of sex reversal as the levels of the Sertoli cell markers; Sox9 and Dmrtl were identical between the WT and mutant XX gonads while their levels were significantly high in the WT XY gonads. These results imply that although 
Lhx2 is expressed in a female dominant manner, is not involved in somatic cell sex determination (or pre-granulosa cell specification) in the developing gonads.

To determine the roles of $L h x 2$, we next carried out RNAseq of the WT and $L h x 2^{\text {flox/flox }}$ ovaries at E12.5 and identified a large number of genes that were differentially expressed. GSEA revealed that the biological processes associated with synaptic transmission, sensory perception, and protein localization to cilium were enriched in $L h x 2^{\text {flox/flox }}$ ovaries. In the brain, Lhx2 is involved in the development and patterning of the neurons involved in sensory perception (Pal et al., 2021; Zembrzycki et al., 2015). In hypothalamic tanycytes, Lhx2 regulates the numbers of motile cilia by controlling the expression of FoxJ1 (Salvatierra et al., 2014). One of the enriched biological processes in $L h x 2^{\text {flox/flox }}$ ovaries was that of hepatobiliary development. Interestingly, Lhx2 regulates the differentiation and activation of hepatic stellate cells, and loss of Lhx2 causes hepatic cell fibrosis (Miyoshi et al., 2019; Wandzioch et al., 2004). Amongst the enriched molecular function in the Lhx2 knockout ovaries was that of $\beta$-Catenin binding. In the developing brain (Hsu et al., 2015; Shetty et al., 2013), and nasopharyngeal carcinoma (Liang et al., 2019) Lhx2 cooperates with $\beta$-Catenin to regulate the expression of its downstream genes. These results imply that Lhx2 has a role in diverse biological processes in multiple tissues, some of which also overlap in the developing ovaries. It appears that some of the functions of $L h x 2$ may be conserved and these commonalities could aid in dissecting its downstream targets or mechanisms that may also be shared between these systems.

Amongst the overrepresented pathways enriched in $L h x 2^{\text {flox/flox }}$ ovaries was that of angiogenesis. In addition, VEGF and PDGF signaling pathways, EGF receptors, and Wnt signaling pathways that are known to aid in vascularization (Gowdak and Krieger, 2018; Gu et al., 2021; Omorphos et al., 2021) were also enriched in the Lhx2 knockout XX gonads. These observations prompted us to ask if angiogenesis was altered in the absence of Lhx2 in the developing ovaries. Towards this, we first tested if angiogenesis has occurred in the developing XX gonads in absence of Lhx2. The results revealed that almost $13 \%$ of the endothelial cell-enriched genes were differentially expressed and most were upregulated upon loss of Lhx2, the mRNA of endothelial cell-enriched genes Jag1 and Pdgfra were upregulated, the transcription factor Tek which is specific to endothelial cells was increased by almost two folds in Lhx2-/- ovaries as compared to WT controls. Also, there were high numbers of VE-Cadherin positive endothelial cells observed in Lhx2-/- ovaries. These results 
imply that loss of $L h x 2$ leads to increased numbers of endothelial cells in the XX gonads. In breast cancer, overexpression of LHX2 is reported to promote tumor growth by controlling vascularization (Kuzmanov et al., 2014). Thus, the requirements of LHX2 in control of vascularization and/or angiogenesis may be context dependent.

We next determined the cell types that expressed Lhx2 in the developing mouse ovaries. Based on the phenotypes observed, we suspected that Lhx2 might be expressed by the endothelial cells and its loss might activate their migration. However, single-cell RNAseq analysis revealed that $L h x 2$ is exclusively expressed in the germ cells of the developing ovaries with no expression in the endothelial cells. This prompted us to hypothesize that LHX2 in a cell non-autonomous manner must regulate endothelial cell migration. In the developing testis, there is a breakdown of mesonephric vascular plexus and endothelial cells from the mesonephros migrate into the gonads leading to the establishment of angiogenesis and the formation of a coelomic blood vessel. In contrast, the mesonephric vascular plexus remains intact and there is no migration of endothelial cells into the developing ovaries until E18.5 (Coveney et al., 2008; Gu et al., 2021; Kumar and DeFalco, 2018). Thus to test if the loss of Lhx2 in the germ cells causes endothelial cell migration, we carried out gonad recombination assays where mesonephros (expressing GFP) from wild type animals was cocultured with the gonads from wild type or Lhx2 knockout mice and tested for the migration of GFP positive cells in the gonadal region. Previous studies have established that in such gonad recombination assay, the migrating cells are endothelial in origin (Brennan et al., 2002; Capel and Batchvarov, 2008; Coveney et al., 2008). We observed that the GFP-positive endothelial cells from the WT mesonephros migrated into the Lhx2-/- ovaries; no such migration was observed in WT XX gonads. Thus, Lhx2 is essential for suppressing endothelial cell migration and preventing vascularization in the ovaries. Using the gonad recombination assay, a study had shown that meiotic germ cells suppressed mesonephric cell migration in the developing ovary in vitro (Yao et al., 2003). However, an in vivo proof for such an event to occur was lacking. To the best of our knowledge, our study provides the first in vivo evidence on the role of germ cells in the control of endothelial cell migration.

Since the loss of $L h x 2$ leads to angiogenesis in the ovary by a cell non-autonomous control, we next aimed to identify the factors that might promote this process. Previous studies have shown that Wnt4, Rspol, and their downstream effector $\beta$-catenin are essential to suppress vascularization in XX gonads (Chassot et al., 2014). Further, GSEA revealed that the Wnt 
pathway and $\square$-catenin binding is overrepresented in the $L h x 2^{\text {flox/flox }} \mathrm{XX}$ gonads. Thus we hypothesized that the ectopic migration of endothelial cells in the XX gonads lacking $L h x 2$ could be due to loss of Wnt4, Rspol, or $\square$-catenin. However, RNAseq data at E12.5 and qPCR analysis at E13.5 revealed that the levels of Wnt4, Rspol, and Ctnnb1 mRNA are identical in the Lhx2-/- and WT ovaries. These results imply that the migration of endothelial cells in the Lhx2-/- XX gonad is not due to loss of Wnt4, Rspol, or $\beta$-catenin. This is not completely unexpected as Wnt4 and Rspolare expressed by the somatic cells of the gonads while $L h x 2$ is in the germ cells. Thus, Lhx2 independent of Wnt/ $\beta$-catenin signaling pathways suppresses angiogenesis in the developing fetal ovaries. Indeed, the expression of Lhx2 is unaltered in developing ovaries knockout for Wnt4 (Supplementary Fig 3F). Thus it appears that both Lhx2 in germ cells and Wnt4 in somatic cells independently operate to suppress angiogenesis in the developing ovaries.

In the developing testis, several molecular pathways and cellular players are implicated in vascular development. It is speculated that in the developing ovary, long-range factors secreted by the meiotic germ cells in the XX gonads suppress mesonephric cell migration (Yao et al., 2003). However, the identity of such a factor(s) is yet not known. In the developing testis, loss of Vegf or blocking of Vegfr2 completely prevents endothelial cell migration (Cool et al., 2011). Interestingly, disruption of PDGF does not block endothelial cell migration; though it does suppress vascular branching in the developing testis (Cool et al., 2011). Interestingly, in breast cancer cells, LHX2 directly controls the expression of $P d g f b$ by binding to its promoter and controlling endothelial cell migration (Kuzmanov et al., 2014). Beyond VEGF and PDGF, the members of EGF, FGF (Fgfl and Fgf2), Inhibin and angiopoietin, and the ephrin family are also known to activate angiogenesis in multiple tissues (Gowdak and Krieger, 2018; Gu et al., 2021; Omorphos et al., 2021; Yao et al., 2006). To determine if the migration of endothelial cells in the ovaries in absence of $L h x 2$ is due to altered expression of these pro-angiogenic factors, we mined the RNAseq data and observed that there is a significant increase in expression of Vegfa, inhibins, Fgfl, and Fgf2 in the Lhx2 knockout ovaries and their levels are similar or even surpassing to those of the WT testis. As compared to ovaries, several angiogenesis-related genes such as angiopoietins are expected to be downregulated in the testis. Interestingly, these were also downregulated in the Lhx2-/ovaries. Overall in the context of the genes that control endothelial cell migration and angiogenesis, Lhx2 knockout XX gonads had an expression profile similar to that of the WT testis. Interestingly, we found that amongst these pro-angiogenic factors, $P d g f a$ and $E g f$ are 
expressed by the germ cell in the XX gonads. At present, it is unknown if the increased expression of pro-angiogenic factors in absence of $L h x 2$ is due to their altered levels in the germ cells. Nevertheless, our results indicate that in the absence of $L h x 2$, migration of endothelial cells in the developing ovaries is non-autonomously controlled by the germ cells creating an intra-gonadal milieu that is pro-angiogenic similar to that of the testis.

According to the current concepts of sex determination, somatic cells initially take a sexspecific fate which is then relayed to other cells in the developing gonads. The process of vascularization is also thought to be a consequence of such sex-specific fate decisions of the somatic cells to the Sertoli cells. This is evident from the fact that, in the Wnt4 and Rspol knockout gonads, a subset of somatic cells overexpresses Sertoli cell markers indicative of somatic sex reversal and there is ectopic vascularization (Chassot et al., 2014; Ungewitter and Yao, 2013). However, we did not observe any over-expression of Sertoli cell-specific genes in the Lhx2-/- XX gonads while there was mesonephric migration of endothelial cells. These observations indicate that sexual dimorphism of the somatic cells and endothelial cells could be independently governed events in the developing gonads. Although the supporting lineage of the Lhx2 knockout ovaries is not masculinized, it will be of interest to investigate the effects of loss of $L h x 2$ on other genes in the somatic and interstitial lineage to investigate the roles of germ cells in somatic cell programming.

It is known that the migrating endothelial cells are capable of developing a vascular network in the developing testis, while the endothelial cells in the developing ovaries do not develop a vascular network (Coveney et al., 2008). Intriguingly, this ability of the endothelial cells to form a vascular network in the two sexes is associated with the differential expression of several (445) genes in these cells. Microarray analysis has identified a sex-biased expression of several genes in the endothelial cells isolated from XX and XY gonads (Jameson et al., 2012). To determine if the transcriptome changes in the endothelial cells in the XX gonads of Lhx2-/- mice, we profiled the expression of endothelial cell-enriched genes in the WT XX, WT XY, and Lhx2-/- XX gonads. As expected, in the control animals the expression of a proportion of endothelial genes was sex-biased with higher levels in the XY gonads as compared to the XX gonads at E12.5. We next compared the expression of all these genes in $L h x 2^{\text {flox/flox }}$ ovaries and observed that in the XX gonads of $L h x 2$ knockout embryos, a reasonable proportion of endothelial cell-enriched genes had a male-biased pattern of expression. For example, Cavl is expressed at higher levels in endothelial cells of the 
developing ovaries as compared to the testis (Bullejos et al., 2002; Piprek et al., 2017), the expression of Cavl was reduced in the Lhx2 knockout XX gonads at levels almost similar to those of the testis. In contrast, Esml is an endothelial cell-specific gene overexpressed in the XY gonads as compared to XX gonads (Jameson et al., 2012). Our results showed that in Lhx2 knockout XX ovaries, Esml was elevated similar to those of the testis (Supplementary Fig 5). These results suggest that in absence of Lhx2 in the developing ovaries, the endothelial cells have a gene expression profile like that in the developing testis. Whether this male-like expression of the endothelial cell-enriched genes in the developing ovaries of Lhx2 mutants is due to overexpression of the pro-angiogenic factor or is induced by other factors needs to be determined.

In the developing testis, the endothelial cells have a restrictive path of migration and form a distinct male-specific vascular pattern that is not detected in the ovaries. Time-lapse, as well as static imaging studies, have shown that in the $\mathrm{XX}$ gonads, individual endothelial cells undergo active movements, and few endothelial cells even venture into the coelomic domain but fail to establish any recognizable coherent structures (Coveney et al., 2008). In contrast, in the XY gonads, the endothelial cell migration is directed toward the coelomic domain where cells aggregate to form the coelomic vessel (Coveney et al., 2008). We tested if the endothelial cells that have migrated in the $L h \times 2-/-\mathrm{XX}$ gonads can develop a vascular pattern similar to that of XY gonads. Towards this, we extended the gonad recombination assays until $72 \mathrm{~h}$ that allowed the endothelial cells to form a distinct network in the XY gonads. We then quantified the length and branching of GFP-positive cells that accumulated in the gonads of both WT testis and Lhx2-/- ovaries. As expected, GFP-positive cells in the developing testis formed a network resembling a microvasculature. Quantitatively there were distinct master segments, branches, junctions, nodes, and that ended in twigs. No such microvascular network was detected in the WT XX gonads except for a few isolated segments (not shown). Interestingly, akin to the testis, GFP positive WT cells were seen to coalesce in the Lhx2-/ovaries and developed a vascular network. Quantitatively, the numbers of master segments and segments representing the vasculature networks were comparable in the Lhx2-/- XX gonads and the WT testis. Albeit, in the XX Lhx2 knockouts, the numbers of isolated segments were lower whereas the number of twigs, branches, junctions, and nodes were elevated as compared to the WT testis suggesting that vasculature is established in the ovaries in absence of $L h x 2$; the pattern might be slightly different as compared to the male gonads. These results imply that in the absence of $L h x 2$, the migrating endothelial cells in the XX 
gonads, to a certain extent have an ability to pattern the vasculature similar to that in the testis.

In the developing testis, the vascular endothelial cell network culminates into a single coelomic blood vessel that lines the ventral aspect of the gonad. Detailed imaging experiments have shown that endothelial cells that detach from mesonephric vascular plexus actively migrate through the testis where they rearrange to form a blood vessel subjacent to the coelomic epithelium (Coveney et al., 2008). In the case of Lhx2-/- ovaries, although there was a migration of cells that organized into a capillary network similar to that of the testis, we failed to detect a coelomic vessel at E13.5. This was not due to a delay in the organization, as even at E15.5 when the testis had a well-patterned vascularization, the endothelial cell that migrated into the $L h \times 2-/-$ ovaries failed to assemble into a coelomic blood vessel. Instead, we observed hemorrhagic areas due to the accumulation of blood in the Lhx2-/- ovaries. To determine why the coelomic blood vessel failed to form in the Lhx2-/- ovaries, we analyzed the RNAseq data and observed that although there is a significant increase in the expression of most angiogenesis factors, expression of $P d g f a$ and $P d g f b$ which are crucial for branching morphogenesis in the developing testis (Cool et al., 2011) were not elevated in the Lhx2-/ovaries to the levels seen in the testis. Thus, the failure to form a coelomic blood vessel could be due to an altered ratio of PDGF and VEGF in the Lhx2-/- ovaries. It will be of interest to study the effects of supplementation and suppression of these factors to prove this speculation.

\section{Conclusion}

To date, the supporting lineage and the interstitial lineage were thought to control vascularization in the developing testis and/or its suppression in the developing ovaries. Our results have hitherto identified the involvement of the germ cells in this control. We show that $L h x 2$ in the germ cell of the developing XX gonads creates an intragonadal milieu that suppresses vascularization. We believe that the knowledge generated through such studies in the long term will be useful in the development of management strategies for disorders of sex development and infertility.

\section{Funding and Acknowledgement}

We thank Prof. Shubha Tole, TIFR for providing $L h x 2-/-$ and $L h x 2^{\text {flox }}$ mice. The project was funded by the Department of Biotechnology (DBT) Government of India under the Grant ID: 
BT/PR10368/MED/97/223/2014 to DM. The DM lab is also funded by grants from the Indian Council of Medical Research (ICMR) Government of India. NS is thankful to ICMR-Senior Research Fellowship. DS is thankful to UGC for the Junior Research Fellowship and AB is a recipient of a Junior Research Fellowship from the DBT project. MKJ was supported by the Ramanujan Fellowship awarded by the Science and Engineering Research Board (SERB), Department of Science and Technology (DST), Government of India (SB/S2/RJN-049/2018). The manuscript bears the NIRRH ID: RA/1123/09-2021.

\section{Conflict of Interest/ Declaration of interest}

The authors declare no conflict of interest. 


\section{References}

Ashary, N., Bhide, A., Chakraborty, P., Colaco, S., Mishra, A., Chhabria, K., Jolly, M.K., Modi, D., 2020. Single-Cell RNA-seq Identifies Cell Subsets in Human Placenta That Highly Expresses Factors Driving Pathogenesis of SARS-CoV-2. Front. Cell Dev. Biol. 8, 1-16. https://doi.org/10.3389/fcell.2020.00783

Bhattacharya, I., Modi, D., 2021. Sex Determination in Teleost Fish, in: Recent Updates in Molecular Endocrinology and Reproductive Physiology of Fish. Springer Singapore, pp. 121-138. https://doi.org/10.1007/978-981-15-8369-8_9

Birk, O.S., Caslano, D.E., Wassif, C.A., Cogilatl, T., Zhaos, L., Zhao, Y., Grinberg, A., Huang, S.P., Kreidberg, J.A., Parker, K.L., Porter, F.D., Westphal, H., 2000. The LIM homeobox gene Lhx9 is essential for mouse gonad formation. Nature 403, 909-913. https://doi.org/10.1038/35002622

Brennan, J., Karl, J., Capel, B., 2002. Divergent vascular mechanisms downstream of sry establish the arterial system in the XY gonad. Dev. Biol. 244, 418-428. https://doi.org/10.1006/dbio.2002.0578

Bulchand, S., Subramanian, L., Tole, S., 2003. Dynamic spatiotemporal expression of LIM genes and cofactors in the embryonic and postnatal cerebral cortex. Dev. Dyn. 226, 460469. https://doi.org/10.1002/DVDY.10235

Bullejos, M., Bowles, J., Koopman, P., 2002. Extensive vascularization of developing mouse ovaries revealed by caveolin-1 expression. Dev. Dyn. 225, 95-99. https://doi.org/10.1002/DVDY.10128

Capel, B., 2017. Vertebrate sex determination: Evolutionary plasticity of a fundamental switch. Nat. Rev. Genet. https://doi.org/10.1038/nrg.2017.60

Capel, B., Batchvarov, J., 2008. Preparing recombinant gonad organ cultures. Cold Spring Harb. Protoc. 3. https://doi.org/10.1101/pdb.prot5078

Cardoso-Moreira, M., Halbert, J., Valloton, D., Velten, B., Chen, C., Shao, Y., Liechti, A., Ascenção, K., Rummel, C., Ovchinnikova, S., Mazin, P. V., Xenarios, I., Harshman, K., Mort, M., Cooper, D.N., Sandi, C., Soares, M.J., Ferreira, P.G., Afonso, S., Carneiro, M., Turner, J.M.A., VandeBerg, J.L., Fallahshahroudi, A., Jensen, P., Behr, R., Lisgo, 
S., Lindsay, S., Khaitovich, P., Huber, W., Baker, J., Anders, S., Zhang, Y.E.,

Kaessmann, H., 2019. Gene expression across mammalian organ development. Nature 571, 505-509. https://doi.org/10.1038/s41586-019-1338-5

Carpentier, G., Berndt, S., Ferratge, S., Rasband, W., Cuendet, M., Uzan, G., Albanese, P., 2020. Angiogenesis Analyzer for ImageJ - A comparative morphometric analysis of "Endothelial Tube Formation Assay" and "Fibrin Bead Assay." Sci. Rep. 10, 1-13. https://doi.org/10.1038/s41598-020-67289-8

Chassot, A.A., Gillot, I., Chaboissier, M.C., 2014. R-spondin1, WNT4, and the ctnnb1 signaling pathway: Strict control over ovarian differentiation. Reproduction. https://doi.org/10.1530/REP-14-0177

Choi, Y., Ballow, D.J., Xin, Y., Rajkovic, A., 2008. Lim homeobox gene, Lhx8, is essential for mouse oocyte differentiation and survival. Biol. Reprod. 79, 442-449. https://doi.org/10.1095/biolreprod.108.069393

Chou, S.J., Tole, S., 2019. Lhx2, an evolutionarily conserved, multifunctional regulator of forebrain development. Brain Res. https://doi.org/10.1016/j.brainres.2018.02.046

Chue, J., Smith, C.A., 2011. Sex determination and sexual differentiation in the avian model. FEBS J. 278, 1027-1034. https://doi.org/10.1111/J.1742-4658.2011.08032.X

Clapcote, S.J., Roder, J.C., 2005. Simplex PCR assay for sex determination in mice. Biotechniques 38, 702-706. https://doi.org/10.2144/05385BM05

Colaco, S., Chhabria, K., Singh, D., Bhide, A., Singh, N., Singh, A., Husein, A., Mishra, A., Sharma, R., Ashary, N., Modi, D., 2021. Expression map of entry receptors and infectivity factors for pan-coronaviruses in preimplantation and implantation stage human embryos. J. Assist. Reprod. Genet. 38, 1709-1720. https://doi.org/10.1007/s10815-021-02192-3

Cool, J., DeFalco, T.J., Capel, B., 2011. Vascular-mesenchymal cross-talk through Vegf and Pdgf drives organ patterning. Proc. Natl. Acad. Sci. U. S. A. 108, 167-172. https://doi.org/10.1073/pnas.1010299108

Coveney, D., Cool, J., Oliver, T., Capel, B., 2008. Four-dimensional analysis of 
vascularization during primary development of an organ, the gonad. Proc. Natl. Acad.

Sci. U. S. A. 105, 7212-7217. https://doi.org/10.1073/pnas.0707674105

Czerwinski, M., Natarajan, A., Barske, L., Looger, L.L., Capel, B., 2016. A timecourse analysis of systemic and gonadal effects of temperature on sexual development of the red-eared slider turtle Trachemys scripta elegans. Dev. Biol. 420, 166-177. https://doi.org/10.1016/j.ydbio.2016.09.018

Ge, W., Wang, J.J., Zhang, R.Q., Tan, S.J., Zhang, F.L., Liu, W.X., Li, L., Sun, X.F., Cheng, S.F., Dyce, P.W., De Felici, M., Shen, W., 2021. Dissecting the initiation of female meiosis in the mouse at single-cell resolution. Cell. Mol. Life Sci. 78, 695-713. https://doi.org/10.1007/s00018-020-03533-8

Godbole, G., Modi, D., 2010. Regulation of decidualization, interleukin-11 and interleukin15 by homeobox A 10 in endometrial stromal cells. J. Reprod. Immunol. 85, 130-139. https://doi.org/10.1016/j.jri.2010.03.003

Godbole, G., Suman, P., Malik, A., Galvankar, M., Joshi, N., Fazleabas, A., Gupta, S.K.S.K., Modi, D., 2017. Decrease in Expression of HOXA10 in the Decidua After Embryo Implantation Promotes Trophoblast Invasion. Endocrinology 158, 2618-2633. https://doi.org/10.1210/en.2017-00032

Gowdak, L.H.W., Krieger, J.E., 2018. Vascular Growth Factors, Progenitor Cells, and Angiogenesis, in: Endothelium and Cardiovascular Diseases: Vascular Biology and Clinical Syndromes. Academic Press, pp. 49-62. https://doi.org/10.1016/B978-0-12812348-5.00005-2

Gu, X., Li, S.Y., DeFalco, T., 2021. Immune and vascular contributions to organogenesis of the testis and ovary. FEBS J. https://doi.org/10.1111/febs.15848

Hsu, L.C.-L., Nam, S., Cui, Y., Chang, C.-P., Wang, C.-F., Kuo, H.-C., Touboul, J.D., Chou, S.-J., 2015. Lhx2 regulates the timing of $\beta$-catenin-dependent cortical neurogenesis. Proc. Natl. Acad. Sci. 112, 12199-12204. https://doi.org/10.1073/PNAS.1507145112

Huang, C.C., Orvis, G.D., Kwan, K.M., Behringer, R.R., 2014. Lhx1 is required in Müllerian duct epithelium for uterine development. Dev. Biol. 389, 124-136. https://doi.org/10.1016/j.ydbio.2014.01.025 
Hunter, C.S., Rhodes, S.J., 2005. LIM-homeodomain genes in mammalian development and human disease. Mol. Biol. Rep. https://doi.org/10.1007/s11033-004-7657-z

Jameson, S.A., Natarajan, A., Cool, J., DeFalco, T., Maatouk, D.M., Mork, L., Munger, S.C., Capel, B., 2012. Temporal transcriptional profiling of somatic and germ cells reveals biased lineage priming of sexual fate in the fetal mouse gonad. PLoS Genet. 8. https://doi.org/10.1371/journal.pgen.1002575

Katsura, Y., Kondo, H.X., Ryan, J., Harley, V., Satta, Y., 2018. The evolutionary process of mammalian sex determination genes focusing on marsupial SRYs. BMC Evol. Biol. 18, 1-11. https://doi.org/10.1186/s12862-018-1119-z

Kumar, D.L., DeFalco, T., 2018. A perivascular niche for multipotent progenitors in the fetal testis. Nat. Commun. 9. https://doi.org/10.1038/s41467-018-06996-3

Kuzmanov, A., Hopfer, U., Marti, P., Meyer-Schaller, N., Yilmaz, M., Christofori, G., 2014. LIM-homeobox gene 2 promotes tumor growth and metastasis by inducing autocrine and paracrine PDGF-B signaling. Mol. Oncol. 8, 401-416. https://doi.org/10.1016/J.MOLONC.2013.12.009

Lecluze, E., Rolland, A.D., Filis, P., Evrard, B., Leverrier-Penna, S., Maamar, M. Ben, Coiffec, I., Lavoué, V., Fowler, P.A., Mazaud-Guittot, S., Jégou, B., Chalmel, F., 2020. Dynamics of the transcriptional landscape during human fetal testis and ovary development. Hum. Reprod. 35, 1099-1119. https://doi.org/10.1093/humrep/deaa041

Li, S.-Y., Gu, X., Heinrich, A., Hurley, E.G., Capel, B., DeFalco, T., 2021. Loss of Mafb and Maf distorts myeloid cell ratios and disrupts fetal mouse testis vascularization and organogenesis $\dagger$. Biol. Reprod. https://doi.org/10.1093/biolre/ioab098

Li, Y., Ray, D., Ye, P., 2013. Identification of germ cell-specific genes in mammalian meiotic prophase. BMC Bioinformatics 14, 72. https://doi.org/10.1186/1471-2105-14-72

Liang, T.S., Zheng, Y.J., Wang, J., Zhao, J.Y., Yang, D.K., Liu, Z.S., 2019. MicroRNA-506 inhibits tumor growth and metastasis in nasopharyngeal carcinoma through the inactivation of the Wnt/ $\beta$-catenin signaling pathway by down-regulating LHX2. J. Exp. Clin. Cancer Res. 38, 1-20. https://doi.org/10.1186/s13046-019-1023-4 
Mangale, V.S., Hirokawa, K.E., Satyaki, P.R.V., Gokulchandran, N., Chikbire, S., Subramanian, L., Shetty, A.S., Martynoga, B., Paul, J., Mai, M. V., Li, Y., Flanagan, L.A., Tole, S., Monuki, E.S., 2008. Lhx2 selector activity specifies cortical identity and suppresses hippocampal organizer fate. Science (80-. ). 319, 304-309. https://doi.org/10.1126/science.1151695

Mishra, A., Galvankar, M., Singh, N., Modi, D., 2020a. Spatial and temporal changes in the expression of steroid hormone receptors in mouse model of endometriosis. J. Assist. Reprod. Genet. 37, 1069-1081. https://doi.org/10.1007/s10815-020-01725-6

Mishra, A., Galvankar, M., Vaidya, S., Chaudhari, U., Modi, D., 2020b. Mouse model for endometriosis is characterized by proliferation and inflammation but not epithelial-tomesenchymal transition and fibrosis. J. Biosci. 45, 105. https://doi.org/10.1007/s12038020-00073-y

Miyoshi, M., Kakinuma, S., Kamiya, A., Tsunoda, T., Tsuchiya, J., Sato, A., Kaneko, S., Nitta, S., Kawai-Kitahata, F., Murakawa, M., Itsui, Y., Nakagawa, M., Azuma, S., Nakauchi, H., Asahina, Y., Watanabe, M., 2019. LIM homeobox 2 promotes interaction between human iPS-derived hepatic progenitors and iPS-derived hepatic stellate-like cells. Sci. Reports 201991 9, 1-13. https://doi.org/10.1038/s41598-018-37430-9

Naillat, F., Yan, W., Karjalainen, R., Liakhovitskaia, A., Samoylenko, A., Xu, Q., Sun, Z., Shen, B., Medvinsky, A., Quaggin, S., Vainio, S.J., 2015. Identification of the genes regulated by Wnt-4, a critical signal for commitment of the ovary. Exp. Cell Res. 332, 163-178. https://doi.org/10.1016/j.yexcr.2015.01.010

Nef, S., Stévant, I., Greenfield, A., 2019. Characterizing the bipotential mammalian gonad, in: Current Topics in Developmental Biology. Curr Top Dev Biol, pp. 167-194. https://doi.org/10.1016/bs.ctdb.2019.01.002

Nicol, B., Yao, H.H.-C., 2015. Gonadal identity in the absence of pro-testis factor SOX9 and pro-ovary factor beta-catenin in mice. Biol. Reprod. 93, 1-12. https://doi.org/10.1095/biolreprod.115.131276

Omorphos, N.P., Gao, C., Tan, S.S., Sangha, M.S., 2021. Understanding angiogenesis and the role of angiogenic growth factors in the vascularisation of engineered tissues. Mol. Biol. 
Rep. 48, 941-950. https://doi.org/10.1007/s11033-020-06108-9

Ortega, E.A., Salvador, Q., Fernandez, M., Ward, M.A., 2019. Alterations of sex determination pathways in the genital ridges of males with limited y chromosome genes. Biol. Reprod. 100, 810-823. https://doi.org/10.1093/biolre/ioy218

Pal, S., Dwivedi, D., Pramanik, T., Godbole, G., Iwasato, T., Jabaudon, D., Bhalla, U.S., Tole, S., 2021. An early cortical progenitor-specific mechanism regulates thalamocortical innervation. J. Neurosci. 41, 6822-6835. https://doi.org/10.1523/JNEUROSCI.0226-21.2021

Piprek, R.P., Kolasa, M., Podkowa, D., Kloc, M., Kubiak, J.Z., 2017. Cell adhesion molecules expression pattern indicates that somatic cells arbitrate gonadal sex of differentiating bipotential fetal mouse gonad. Mech. Dev. 147, 17-27. https://doi.org/10.1016/j.mod.2017.07.001

Porter, F.D., Drago, J., Xu, Y., Cheema, S.S., Wassif, C., Huang, S.P., Lee, E., Grinberg, A., Massalas, J.S., Bodine, D., Alt, F., Westphal, H., 1997. Lhx2, a LIM homeobox gene, is required for eye, forebrain, and definitive erythrocyte development. Development 124, 2935-2944. https://doi.org/10.1242/dev.124.15.2935

Rotgers, E., Jørgensen, A., Yao, H.H.C., 2018. At the crossroads of fate-Somatic cell lineage specification in the fetal gonad. Endocr. Rev. https://doi.org/10.1210/er.2018-00010

Salvatierra, J., Lee, D.A., Zibetti, C., Duran-Moreno, M., Yoo, S., Newman, E.A., Wang, H., Bedont, J.L., De Melo, J., Miranda-Angulo, A.L., Gil-Perotin, S., Garcia-Verdugo, J.M., Blackshaw, S., 2014. The LIM homeodomain factor Lhx2 is required for hypothalamic tanycyte specification and differentiation. J. Neurosci. 34, 16809-16820. https://doi.org/10.1523/JNEUROSCI.1711-14.2014

Shetty, A.S., Godbole, G., Maheshwari, U., Padmanabhan, H., Chaudhary, R., Muralidharan, B., Hou, P.S., Monuki, E.S., Kuo, H.C., Rema, V., Tole, S., 2013. Lhx2 regulates a cortex-specific mechanism for barrel formation. Proc. Natl. Acad. Sci. U. S. A. 110. https://doi.org/10.1073/pnas.1311158110

Singh, N., Modi, D., 2020. The Molecular Genetics of Testis Determination, in: Genetics of Male Infertility. Springer, Cham, pp. 3-17. https://doi.org/10.1007/978-3-030-37972- 


\section{8_1}

Singh, N., Singh, D., Modi, D., 2021. LIM Homeodomain (LIM-HD) Genes and Their CoRegulators in Developing Reproductive System and Disorders of Sex Development. Sex. Dev. 1-15. https://doi.org/10.1159/000518323

Svingen, T., Koopman, P., 2013. Building the mammalian testis: Origins, differentiation, and assembly of the component cell populations. Genes Dev. https://doi.org/10.1101/gad.228080.113

Tang, F., Richardson, N., Albina, A., Chaboissier, M.C., Perea-Gomez, A., 2020. Mouse Gonad Development in the Absence of the Pro-Ovary Factor WNT4 and the Pro-Testis Factor SOX9. Cells 9. https://doi.org/10.3390/cells9051103

Tiwari, A., Ashary, N., Singh, N., Sharma, S., Modi, D., 2021. Modulation of E-Cadherin and N-Cadherin by ovarian steroids and embryonic stimuli. Tissue Cell 73, 101670. https://doi.org/10.1016/J.TICE.2021.101670

Ungewitter, E.K., Yao, H.H.-C., 2013. How to Make a Gonad: Cellular Mechanisms Governing Formation of the Testes and Ovaries. Sex. Dev. 7, 7-20. https://doi.org/10.1159/000338612

van Dijk, D., Sharma, R., Nainys, J., Yim, K., Kathail, P., Carr, A.J., Burdziak, C., Moon, K.R., Chaffer, C.L., Pattabiraman, D., Bierie, B., Mazutis, L., Wolf, G., Krishnaswamy, S., Pe'er, D., 2018. Recovering Gene Interactions from Single-Cell Data Using Data Diffusion. Cell 174, 716-729.e27. https://doi.org/10.1016/J.CELL.2018.05.061/ATTACHMENT/7AC4A2F9-F866-4375960C-C4CEAAC61F07/MMC3.XLSX

Wandzioch, E., Kolterud, Å., Jacobsson, M., Friedman, S.L., Carlsson, L., 2004. Lhx2-/mice develop liver fibrosis. Proc. Natl. Acad. Sci. U. S. A. 101, 16549-16554. https://doi.org/10.1073/pnas.0404678101

Whiteley, S.L., Holleley, C.E., Wagner, S., Blackburn, J., Deveson, I.W., Marshall Graves, J.A., Georges, A., 2021. Two transcriptionally distinct pathways drive female development in a reptile with both genetic and temperature dependent sex determination, PLoS Genetics. https://doi.org/10.1371/journal.pgen.1009465 
Yao, H.H., Dinapoli, L., Capel, B., 2003. Meiotic germ cells antagonize mesonephric cell migration and testis cord formation in mouse gonads 5895-5902. https://doi.org/10.1242/dev.00836

Yao, H.H.C., Aardema, J., Holthusen, K., 2006. Sexually dimorphic regulation of inhibin beta B in establishing gonadal vasculature in mice. Biol. Reprod. 74, 978-983. https://doi.org/10.1095/biolreprod.105.050286

Yasuoka, Y., Taira, M., 2021. LIM homeodomain proteins and associated partners: Then and now, in: Current Topics in Developmental Biology. Curr Top Dev Biol, pp. 113-166. https://doi.org/10.1016/bs.ctdb.2021.04.003

Yildirim, E., Aksoy, S., Onel, T., Yaba, A., 2020. Gonadal development and sex determination in mouse. Reprod. Biol. 20, 115-126. https://doi.org/10.1016/J.REPBIO.2020.01.007

Zembrzycki, A., Perez-Garcia, C.G., Wang, C.F., Chou, S.J., O’Leary, D.D.M., 2015. Postmitotic regulation of sensory area patterning in the mammalian neocortex by Lhx 2 . Proc. Natl. Acad. Sci. U. S. A. 112, 6736-6741.

https://doi.org/10.1073/pnas.1424440112

Zhao, L., Wang, C., Lehman, M.L., He, M., An, J., Svingen, T., Spiller, C.M., Ng, E.T., Nelson, C.C., Koopman, P., 2018. Transcriptomic analysis of mRNA expression and alternative splicing during mouse sex determination. Mol. Cell. Endocrinol. 478, 84-96. https://doi.org/10.1016/j.mce.2018.07.010 